\title{
Evaluation of the control effect of SAR inducers against citrus Huanglongbing applied by foliar spray, soil drench or trunk injection
}

Jinyun Li, Vladimir G. Kolbasov, Zhiqian Pang, Shuo Duan, Donghwan Lee, Yixiao Huang, Jin Xu, Doron Teper, Tirtha Lamichhane and Nian Wang ${ }^{*}$ (D)

\begin{abstract}
Huanglongbing (HLB) or greening disease, associated with the bacterial pathogen Candidatus Liberibacter asiaticus (Las), is currently the most devastating citrus disease worldwide and no cure is available. Inducers of systemic acquired resistance (SAR) are effective and sustainable to combat various plant diseases. In this study, the SAR inducers acibenzolar-S-methyl (ASM), imidacloprid (IMI), 2,6-dichloroisonicotinic acid (INA), and salicylic acid (SA), applied individually by foliar spray, soil drench or trunk injection at various rates and frequencies, were evaluated for control of HLB in a 3-year field trial with mature Hamlin sweet orange trees in central Florida, USA in the 2016, 2017, and 2018 crop seasons. Six foliar sprays, six soil applications, and three trunk injections of ASM, IMI, INA, or SA per year were conducted with the untreated as a negative control. HLB disease severity, Las titers, pre-harvest fruit drop, yield and fruit quality were investigated for the treatments. By the end of the 2018 season, all trunk injection treatments at $0.25 \mathrm{~g} /$ tree and foliar sprays of INA or SA (but not ASM or IMI) at $0.5 \mathrm{~g} /$ tree significantly reduced disease severity, Las population, and fruit drop, and increased fruit yield; whereas all foliar spray treatments at $0.25 \mathrm{~g} /$ tree, trunk injection treatments at $0.125 \mathrm{~g} /$ tree, and soil drench treatments at 0.25 or $0.5 \mathrm{~g} /$ tree did not provide effective control of HLB. Additionally, all trunk injection treatments at $0.25 \mathrm{~g} /$ tree had shown a significant decrease in fruit drop and increase of fruit yield starting from 2016 after 1 year of applications, whereas foliar sprays of INA or SA at $0.5 \mathrm{~g} /$ tree exhibited similar effects at 2018 after 3 years of applications. None of the SAR inducer treatments had significant effect on fruit quality. Economic analysis suggested that the trunk injection treatments at $0.25 \mathrm{~g} /$ tree might produce financial benefits. Overall, this study presents useful information for management of citrus HLB with SAR inducers.
\end{abstract}

Keywords: Citrus, Disease control, Huanglongbing (HLB), Induced plant resistance, Liberibacter, Systemic acquired resistance (SAR), Salicylic acid

\section{Background}

Citrus huanglongbing (HLB), also called citrus greening disease, is currently the most destructive citrus disease affecting the global citrus industry and causes significant economic losses worldwide (Blaustein et al. 2018; Wang

\footnotetext{
* Correspondence: nianwang@ufl.edu

Department of Microbiology and Cell Science, Citrus Research and Education Center, Institute of Food and Agricultural Sciences, University of Florida, 700 Experiment Station Road, Lake Alfred, FL 33850, USA
}

2019; Andrade et al. 2020). HLB is associated with the three phloem-colonizing and uncultured bacterial species of 'Candidatus Liberibacter': 'Ca. L. asiaticus' (Las) in Asia, Africa and the Americas, 'Ca. L. africanus' (Laf) in Africa and limited areas of Asia, and ' $\mathrm{Ca}$. L. americanus' (Lam) in Brazil (Bové 2006; Gottwald et al. 2007; Wang 2020). Among them, Las is the most prevalent species worldwide (Bové 2006; Gottwald 2010; Wang et al. 2017b; Andrade et al. 2020). Las is naturally

(c) The Author(s). 2021 Open Access This article is licensed under a Creative Commons Attribution 4.0 International License, which permits use, sharing, adaptation, distribution and reproduction in any medium or format, as long as you give

appropriate credit to the original author(s) and the source, provide a link to the Creative Commons licence, and indicate if changes were made. The images or other third party material in this article are included in the article's Creative Commons licence, unless indicated otherwise in a credit line to the material. If material is not included in the article's Creative Commons licence and your intended use is not permitted by statutory regulation or exceeds the permitted use, you will need to obtain permission directly from the copyright holder. To view a copy of this licence, visit http://creativecommons.org/licenses/by/4.0/ 
transmitted to citrus by the Asian citrus psyllid (ACP, Diaphorina citri Kuwayama) during feeding on citrus plants (Bové 2006). HLB disease symptoms include leaf mottling, yellow shoots, dead and dying twigs, deformed and discolored fruits, premature fruit drop, root decline, and eventually tree mortality (Bové 2006; Gottwald 2010; Johnson et al. 2014). Most commercial citrus varieties are susceptible to Las infection, with few, such as Sugar Belle, showing tolerance to HLB (Clark et al. 2018; Wang 2019; Andrade et al. 2020). Since it was first identified in East Asia over 100 years ago, HLB has spread to most citrus producing areas in the world, including Asia, Africa, and the Americas, except Australia and the Mediterranean region (Wang 2019). In the US, HLB is present in most citrus producing states including the top three producers: Florida, California, and Texas. HLB is endemic in Florida and the estimated losses to the Florida citrus industry caused by HLB exceed $\$ 8$ billion (Singerman and Futch 2018). HLB was first detected in Texas in January 2012 and HLB-positive trees were approximately 38.7\% in 2017 (Sétamou et al. 2020). HLB is also threatening the California citrus industry (Graham et al. 2020) with HLB first reported in a residential property in Hacienda Heights, Los Angeles Co. in March 2012 (Kumagai et al. 2013). Approximately 2000 trees in residential areas have tested positive for Las. Importantly, the first Las-positive $\mathrm{ACP}$ was found in a commercial citrus grove in California in August 2020 (California Citrus Pest and Disease Prevention Program 2020).

Currently, there are no practical cures for HLB (Andrade et al. 2020). Different HLB management strategies are being implemented in different citrus producing areas depending on the overall HLB incidence in the corresponding region. For example in China, a comprehensive HLB control strategy is being used including control of the insect vector ACP, and eradication of symptomatic trees, as well as the use of pathogen-free seedlings for new plantings (Wang et al. 2017a; Blaustein et al. 2018; Andrade et al. 2020). However, in many citrus producing areas where HLB is endemic or has high incidence, such as Florida, inoculum eradication is largely abandoned (Graham et al. 2020). Intriguingly, despite that Las has not been cultured in artificial media, promising advances have been made that might help develop long-term solutions to control HLB, including understanding the virulence mechanisms of Las (Duan et al. 2009; Jain et al. 2018; Pitino et al. 2018; Andrade et al. 2019; Andrade and Wang 2019; Chen et al. 2020; Clark et al. 2020; Pandey et al. 2020; Pang et al. 2020; Thapa et al. 2020a; Thapa et al. 2020b; Vasconcelos et al. 2020), improving Las detection (Kim and Wang 2009; Pagliaccia et al. 2017; Pandey and Wang 2019; Gottwald et al. 2020), and developing tolerant or resistant citrus varieties using traditional and novel approaches (Jia and
Wang 2014; Rawat et al. 2015; Deng et al. 2019; Jia et al. 2019; Jia and Wang 2020). In addition, in HLB-endemic regions, such as Florida, the HLB management focus has shifted to how to improve and sustain the productivity at the presence of HLB. Therefore, multiple approaches were tested to mitigate HLB damage and losses caused by HLB, including maintaining optimal tree growth conditions (Gottwald et al. 2012; Stansly et al. 2014), targeting Las (Akula et al. 2011; Hu and Wang 2016; Barnett et al. 2019; Hussain et al. 2019; Li et al. 2019b), eliciting plant defenses (Zhang et al. 2014; Canales et al. 2016; Li et al. 2016; Li et al. 2017; Hu et al. 2018; Li et al. 2019a), thermotherapy (Thapa et al. 2020a; Thapa et al. 2020b), and biological control (Riera et al. 2017; Wang et al. 2017b; Blaustein et al. 2018).

Induced resistance, either locally or systemically, has been shown to be effective for control of numerous plant diseases (Durrant and Dong 2004; Graham and Myers 2011; Justyna and Ewa 2013; Walters et al. 2013; Pieterse et al. 2014; Riera et al. 2018; Frąckowiak et al. 2019). Specifically, systemic acquired resistance (SAR) has attracted much attention because of its long-lasting control of a broad spectrum of plant diseases (Durrant and Dong 2004). SAR requires specific signaling pathways that involve the signal molecule salicylic acid (SA) and the accumulation of pathogenesis related (PR) proteins (Durrant and Dong 2004). SAR may be activated by plant pathogens or chemical inducers (Walters et al. 2013; Aranega-Bou et al. 2014). Many plant defense inducers, including acibenzolar-S-methyl (ASM), $\beta$-aminobutyric acid (BABA), benzothiadiazole (BTH, synonym of ASM), and 2, 6-dichloroisonicotinic acid (INA), have been evaluated extensively for their efficacy on disease control under natural conditions (Vallad and Goodman 2004; Walters et al. 2013; Li et al. 2016, 2017; Frąckowiak et al. 2019). Numerous earlier studies have shown that SAR inducers provided inconsistent effectiveness in protection against various plant pathogens under field conditions, varying from lack of control to $99 \%$ from field to field, depending on pathogens, host genotypes, nutritional conditions, environmental conditions as well as application time, frequency, and rate, and mode of delivery (Vallad and Goodman 2004; Walters et al. 2013; Li et al. 2016; Frąckowiak et al. 2019). Such relatively large variability in their efficacy underlines the importance of optimizing application methods of plant defense inducers under local environmental conditions.

Several earlier studies have noted that application methods greatly affected plant defense inducers' effectiveness in disease control, with trunk injection and root/soil application superior to foliar spray (Dekkers et al. 2004; Graham and Leite 2004; Francis et al. 2009; Graham and Myers 2011; Aćimović and Meredith 2017). For example, foliar application of ASM was effective against citrus canker under greenhouse conditions but not in field trials 
(Graham and Leite 2004), while soil application of ASM provided effective protection from canker on flushes of young grapefruit trees in the field, despite the efficacy depended on rate, frequency, and timing of application (Graham and Myers 2011). Delivery of the SAR-activator candidate Regalia/MBI-10612 (5 and 12\% extract of plant Reynoutria sachalinensis) into apple plants by trunk injection increased the efficacy in fire blight control in comparison to foliar spray (Aćimović and Meredith 2017). Altogether, these studies indicate that optimizing application methods of plant defense inducers may achieve a better efficacy in disease control.

Previous research has demonstrated that treatment of citrus plants with SAR inducers resulted in enhanced resistance to both bacterial and fungal diseases. For example, soil application of ASM, INA, and imidacloprid (IMI) provided effective control of citrus canker in greenhouse assays (Francis et al. 2009). Soil-applied ASM, IMI, and thiamethoxam provided effective protection from canker on foliar flushes of young citrus trees under epidemic conditions (Graham and Myers 2011, 2013). Foliar spray of citrus with BTH induced resistance to citrus scab, melanose and Alternaria brown spot (Agostini et al. 2003). Furthermore, foliar spray of $\mathrm{BABA}, \mathrm{BTH}$, and INA on citrus, individually or in combination, were effective in slowing down HLB disease progression (Li et al. 2016). Trunk injection of the SAR inducers ASM, SA, oxalic acid, and potassium phosphate significantly suppressed Las titer and disease progress of HLB in the field (Hu et al. 2018). These studies suggest that trunk injections of SAR inducers seem to provide better treatment effects than foliar sprays in the control of citrus HLB. However, to date, no investigations have been conducted to evaluate and compare the efficacy of SAR inducers applied via different methods at a comparable rate and frequency, and consequently, to optimize their application for effective control of citrus HLB.

The purpose of this study was to evaluate the effects of the four SAR inducers ASM, IMI, INA, and SA, applied individually by foliar spray, soil drench, or trunk injection at various rates and frequencies, on HLB disease progression and fruit production under field conditions, to optimize application of SAR inducers for the management of citrus HLB.

\section{Results}

\section{Effect of SAR inducer treatments on HLB disease progression and Las titers}

For all treatments, the HLB symptoms, such as blotchy mottle, loss of foliage, dead and dying twigs, generally became more severe, showing a gradual increase in the HLB severity over time (Fig. 1). However, six treatments showed various effectiveness in reduction of HLB disease severity (Fig. 1).
The HLB disease severity (expressed as standardized area under the disease progress stair, sAUDPS) over the experiment duration was significantly $(P<0.05)$ reduced by foliar sprays of INA $(0.5 \mathrm{~g} /$ tree $)$ and SA $(0.5 \mathrm{~g} /$ tree $)$, and trunk injections of ASM, IMI, INA, or SA at $0.25 \mathrm{~g} /$ tree compared with the untreated control (UTC) (Fig. 2). The Las bacterial titers under these six treatments were also significantly $(P<0.05)$ lower than that of the UTC at the end of the experiments (Table 1). From the initiation to end of the tests, the mean values of Las titer in the INA $(0.5 \mathrm{~g} /$ tree $)$ or SA $(0.5 \mathrm{~g} /$ tree $)$ spray-treated trees increased from $1.21 \times 10^{7}$ and $0.87 \times 10^{7}$ to $3.05 \times 10^{7}$ and $2.12 \times 10^{7}$ cells/g plant tissue, respectively, whereas that of the UTC increased from $0.75 \times 10^{7}$ to $7.63 \times 10^{7}$ cells/g plant tissue. In the ASM, IMI, INA, or SA injection $(0.25 \mathrm{~g} /$ tree $)$-treated trees, Las titers were not significantly altered but maintained a relatively stable level at 1.05 to $1.75 \times 10^{7}$ cells/g plant tissue over time (Table 1 ). These observations indicated that the ASM, IMI, INA, and SA injections at $0.25 \mathrm{~g} /$ tree had better efficacy of suppressing Las growth and HLB disease development than the foliar sprays at $0.5 \mathrm{~g} /$ tree. HLB disease severity was also reduced by foliar sprays of INA ( $0.25 \mathrm{~g} /$ tree) and SA $(0.25$ $\mathrm{g} /$ tree) compared with the UTC, but the differences were not statistically significant $(P>0.05)$ (Fig. 2$)$. The Las titer in the INA $(0.25 \mathrm{~g} /$ tree $)$ - or SA $(0.25 \mathrm{~g} /$ tree $)$-sprayed tree was not significantly $(P>0.05)$ different from that of the UTC over time (Table 1). Applications of the SAR inducers via soil drench at 0.25 or $0.5 \mathrm{~g} /$ tree or via trunk injections at $0.125 \mathrm{~g} /$ tree were not effective in reduction of HLB symptom expressions or Las titer compared with the UTC in this field trial (Figs. 1, 2 and Table 1).

\section{Effect of SAR inducer treatments on fruit drop, yield and quality}

The pre-harvest fruit drop, fruit yield and quality data were collected for all treatments from the beginning (2015) to the end (2018) of the field trial. In September 2017, the hurricane Irma caused severe fruit drop of all trees in this grove, and consequently, no differences in fruit drop or yield were observed among the treatments (Tables 2 and 3). Therefore, the 2017 data were omitted for further discussion.

Prior to the era of HLB, production for Hamlin sweet oranges in Florida on 13-year-old trees averaged more than $112 \mathrm{~kg}$ per tree (Roka et al. 2000). Yields for all treatments during the trial were substantially below the historical level (Table 2). One month after the initiation of treatment applications in 2015, no significant differences $(P>0.05)$ were observed in fruit yield among the treatments including the UTC. But in both 2016 and 2018, the INA $(0.5 \mathrm{~g} /$ tree $)$ or SA ( $0.5 \mathrm{~g} /$ tree $)$ sprays and the ASM, IMI, INA, or SA injections at $0.25 \mathrm{~g} /$ tree resulted in a significantly $(P<0.05)$ higher yield compared 

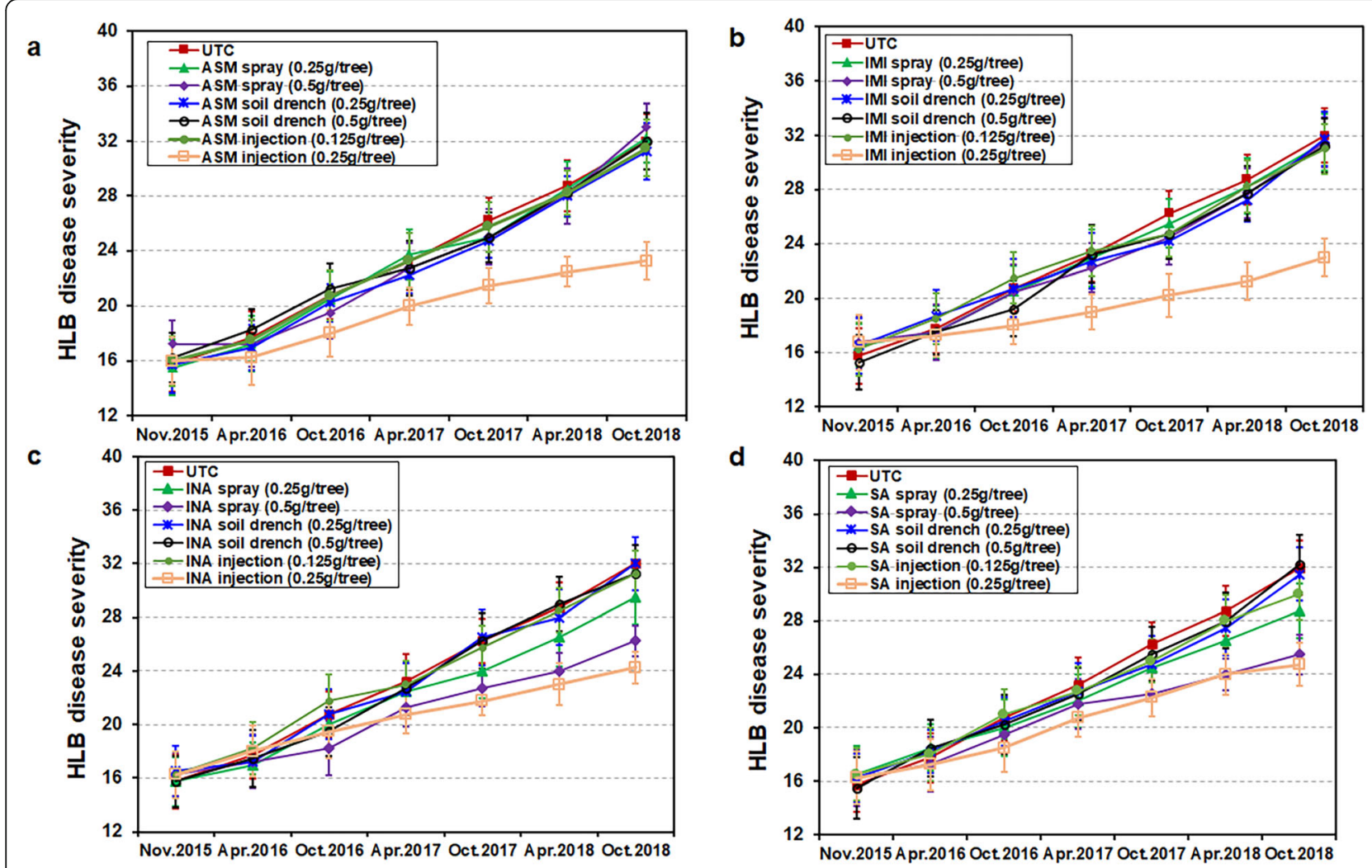

Fig. $1 \mathrm{HLB}$ disease severity progression of Hamlin sweet orange trees following treatments with SAR inducers. a Disease progression under ASM treatments. b Disease progression under IMI treatments. c Disease progression under INA treatments. $\mathbf{d}$ Disease progression under SA treatments. The mean of disease severity was calculated from evaluations of the treated trees. Disease severity scores were shown as means and error bars (standard deviation) $(n=6)$. The untreated control (UTC, spray with water only) was included in each panel for comparison purpose

with the UTC; among these six treatments, there were no significant differences in yield in either 2016 or 2018 (Table 2). The average yield of the six treatments ranged from 52.7 to $60.7 \mathrm{~kg} /$ tree in 2016 and from 60.5 to 63.3 $\mathrm{kg} /$ tree in 2018, whereas that of the UTC was 47.1 and $48.5 \mathrm{~kg} /$ tree in 2016 and 2018, respectively (Table 2).

When compared year by year, the ASM, IMI, INA, or SA injections at $0.25 \mathrm{~g} /$ tree exhibited a significantly $(P<$ 0.05) higher yield in both 2016 and 2018 than 2015 (Table 2), indicating a significant fruit yield increase after 1-year or longer applications; whereas the INA ( 0.5 $\mathrm{g} /$ tree) or SA (0.5 g/tree) sprays showed a significantly higher yield in 2018 (but not in 2016) than in 2015 (Table 2), suggesting a significant yield increase after continuous applications for 3 years.

Similarly, in 2015 , no significant differences $(P>0.05)$ were observed in pre-harvest fruit drop among all treatments including the UTC. But in both 2016 and 2018, the INA $(0.5 \mathrm{~g} /$ tree $)$ or SA $(0.5 \mathrm{~g} /$ tree $)$ sprays and the ASM, IMI, INA, or SA injections at $0.25 \mathrm{~g} /$ tree resulted in a significantly $(P<0.05)$ fewer fruit drop compared with the UTC (Table 3). When compared year by year, the ASM, IMI, INA, or SA injections at $0.25 \mathrm{~g} /$ tree had a significantly lower number of fruit drop in both 2016 and 2018 than 2015 (Table 3), indicating a significant decrease of fruit drop after 1-year or longer applications; whereas the INA $(0.5 \mathrm{~g} /$ tree $)$ or SA $(0.5 \mathrm{~g} /$ tree $)$ sprays showed a significant decrease in fruit drop in 2018 (but not in 2016) than 2015 (Table 3), suggesting that a significant effect on fruit drop after continuous applications for 3 years. These observations demonstrated that the ASM, IMI, INA, or SA injections at $0.25 \mathrm{~g} /$ tree were more effective in reducing pre-harvest fruit drop and sustaining yield than the INA $(0.5 \mathrm{~g} /$ tree $)$ or SA $(0.5 \mathrm{~g} /$ tree) sprays.

Foliar sprays of ASM, IMI, INA, or SA at $0.25 \mathrm{~g} /$ tree, soil applications of the SAR inducers, or trunk injections at $0.125 \mathrm{~g} /$ tree did not show a positive effect on fruit yield (Table 2) or pre-harvest fruit drop (Table 3). In addition, none of the treatments had a statistically significant positive effect on fruit quality, with regard to percent juice content, fruit brix, acidity, or fruit brix acidity ratio; although ASM, IMI, INA, or SA injections at $0.25 \mathrm{~g} /$ tree resulted in a lower acidity and higher brix acidity ratio compared with the UTC, the differences were not statistically significant $(P>0.05)$ (Table 4$)$. 


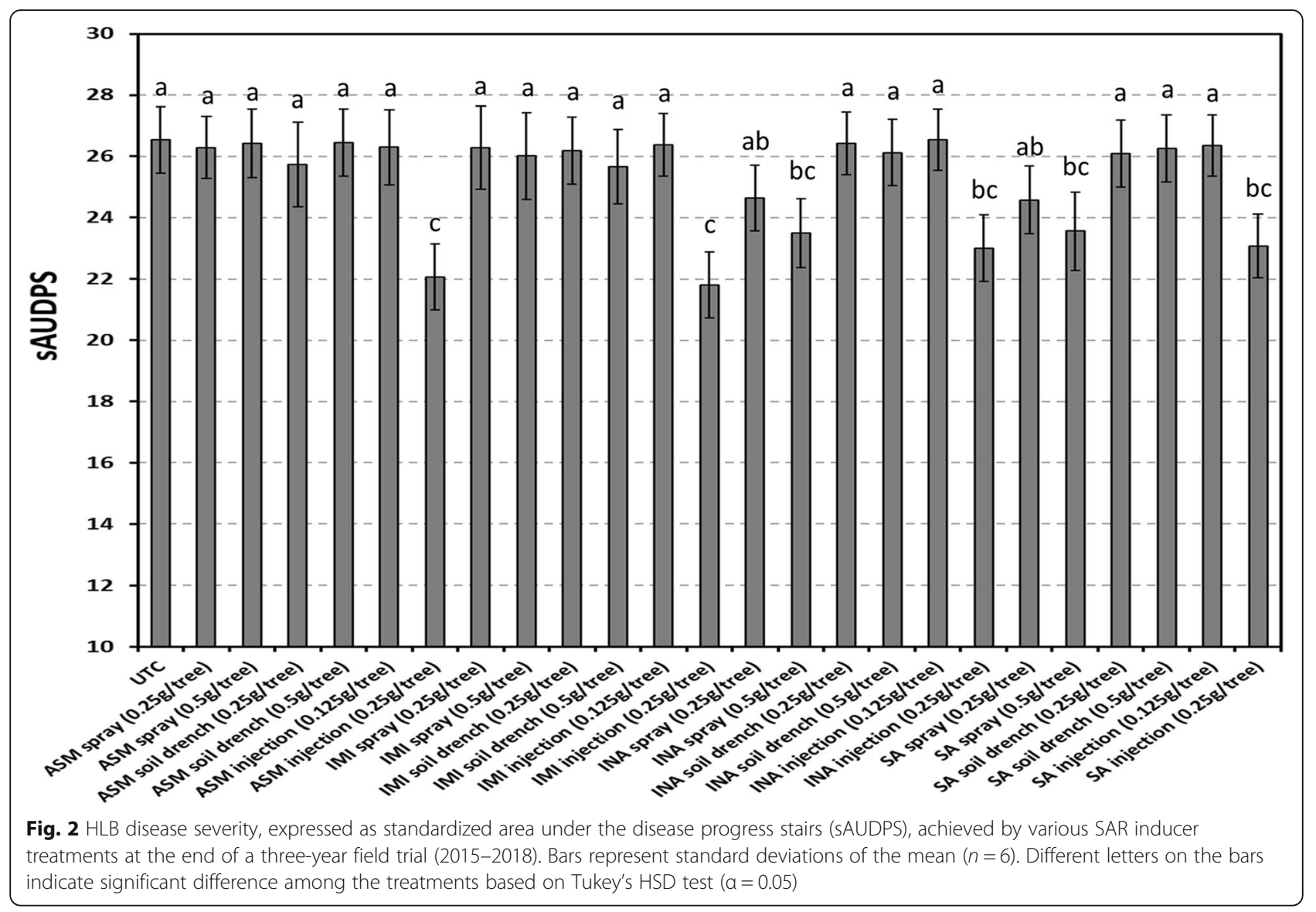

When compared year by year, the ASM, IMI, INA, or SA injections at $0.25 \mathrm{~g} /$ tree had a lower acidity and higher brix acidity ratio in 2016, 2017, and 2018 than 2015 , but the differences were not statistically significant $(P>0.05)$ (Table 4).

\section{Growth performance of citrus treated with SAR inducers}

Following foliar spray, soil application or trunk injection of ASM, IMI, INA or SA during the spring, summer, and fall flushes, all treated trees produced vigorous new shoots with normal leaves. No symptoms of discoloring, stunting and other abnormal appearance were observed on new flushes or shoots in the treated trees over the course of 3 years. These observations indicated that the SAR inducers did not have a phytotoxic effect at the rates tested. However, in 5 of the 48 trees (10.4\%) receiving trunk injection treatments, the wounds caused by drilling holes did not heal completely within 4 months of injection, with cracking in the area around drilling site, indicating certain mechanical injuries to the trunk. These observations suggested that fewer injections per crop season are needed to alleviate the potential harmful effects.

\section{Economic analysis}

Economic feasibility of the six treatments showing improvement of fruit yield, i.e., the INA $(0.5 \mathrm{~g} /$ tree $)$ or SA $(0.5 \mathrm{~g} /$ tree $)$ sprays and the ASM, IMI, INA, or SA injections at $0.25 \mathrm{~g} /$ tree, was evaluated by comparing the revenue with the cost for the 2016 and 2018 growing seasons. Higher yields for treated trees resulted in greater gross values of the fruit harvested from treated trees for all the six treatments in both years (Table 5). The revenue produced from harvested fruit was greater than the estimated costs for ASM, IMI, INA, or SAinjected (at $0.25 \mathrm{~g} /$ tree) trees in both years and improvements in revenue relative to untreated control were observed consistently for these four treatments (Table 5). However, the costs for the INA $(0.5 \mathrm{~g} /$ tree $)$ spray treatment were greater than the revenue generated from harvested fruit in both 2016 and 2018, and no increased revenue was observed for this treatment in either year. Similarly, in 2016, the revenue of the SA $(0.5 \mathrm{~g} /$ tree $)$ spray treatment was offset by the cost and there was no increased revenue for this treatment; whereas, in 2018, the revenue was greater than the cost of the SA $(0.5 \mathrm{~g} /$ tree) spray treatment and increased revenue was observed for the treatment (Table 5). 
Table 1 Candidatus Liberibacter asiaticus (Las) titers in leaf samples of Hamlin sweet orange trees treated with various SAR inducers in a three-year field trial (2015-2018)

\begin{tabular}{|c|c|c|c|c|c|c|c|}
\hline \multirow[t]{2}{*}{ Treatment } & \multicolumn{7}{|c|}{ Las titer $\left(\right.$ Log $_{10}\left[\right.$ cells/g of leaf tissue]) ${ }^{z}$} \\
\hline & Nov.2015 & Apr.2016 & Oct.2016 & Apr.2017 & Oct.2017 & Apr.2018 & Oct.2018 \\
\hline$\overline{\text { UTC }}$ & $6.87 a^{D}$ & $7.16_{a}^{C D}$ & $7.37_{a}^{B C}$ & $7.42 a^{B C}$ & $7.51 \mathrm{a}^{\mathrm{ABC}}$ & $7.64 a^{A B}$ & $7.88 a^{A}$ \\
\hline ASM spray ( $0.25 \mathrm{~g} /$ tree $)$ & $6.92 a^{D}$ & $7.11_{\mathrm{a}} \mathrm{CD}$ & $7.29 a_{a} C D$ & $7.33 a_{a}^{B C}$ & $7.47 \mathrm{a} A B C$ & $7.56 a^{A B}$ & $7.78 \mathrm{ab}^{\mathrm{A}}$ \\
\hline ASM spray (0.5 g/tree) & $6.84 a^{D}$ & $7.06 a_{a}^{C D}$ & $7.15 a_{a}^{C D}$ & $7.30 a^{B C}$ & $7.44 a_{a} A B C$ & $7.67 a_{a}^{A B}$ & $7.76 \mathrm{ab}^{\mathrm{A}}$ \\
\hline ASM soil drench ( $0.25 \mathrm{~g} /$ tree $)$ & $7.07 a^{D}$ & 7.21 a $C D$ & $7.26{ }_{a}^{C D}$ & $7.47 a^{B C}$ & $7.51 \mathrm{a} A B C$ & $7.64 a_{a}^{A B}$ & $7.87 \mathrm{a}^{\mathrm{A}}$ \\
\hline ASM soil drench (0.5 g/tree) & $6.96 a^{D}$ & 7.11 a $C D$ & $7.22 \mathrm{a}^{C D}$ & $7.28 a^{B C}$ & $7.42 a^{A B C}$ & $7.66 a_{a}^{A B}$ & $7.79 a^{A}$ \\
\hline ASM injection (0.125 g/tree) & $6.81_{a}{ }^{C}$ & $7.09 a_{a}^{B C}$ & $7.27 a^{B}$ & $7.39 \mathrm{a}^{\mathrm{B}}$ & $7.55 a_{a}^{A B}$ & $7.71 \mathrm{a}$ & $7.85 a^{A}$ \\
\hline ASM injection ( $0.25 \mathrm{~g} /$ tree $)$ & $7.13 \mathrm{a}^{\mathrm{A}}$ & $7.17 \mathrm{a}^{\mathrm{A}}$ & $7.23 \mathrm{a}^{\mathrm{A}}$ & $7.25 \mathrm{a}^{\mathrm{A}}$ & $7.27 \mathrm{a}^{\mathrm{A}}$ & $7.17 b^{A}$ & $7.21 c^{\mathrm{A}}$ \\
\hline IMI spray (0.25 g/tree) & $6.91 a_{a}{ }^{C}$ & $7.15 a^{B C}$ & $7.22 a^{A B}$ & $7.32 a_{a}^{A B}$ & $7.49 a_{a}^{A B}$ & $7.60 a^{A}$ & $7.78 \mathrm{ab}^{\mathrm{A}}$ \\
\hline IMI spray (0.5 g/tree) & $7.02 a^{C}$ & $7.24 a^{B C}$ & $7.29 a^{B C}$ & $7.35 a^{B C}$ & $7.52 a^{A B}$ & $7.59 a_{a}^{A B}$ & $7.82 \mathrm{a}^{\mathrm{A}}$ \\
\hline IMI soil drench (0.25 g/tree) & $7.11 \mathrm{a}^{\mathrm{D}}$ & $7.19 a^{C D}$ & $7.30 a_{a}^{C D}$ & $7.38 a^{B C}$ & $7.54_{a} A B C$ & $7.68 a^{A B}$ & $7.89 a^{A}$ \\
\hline IMI soil drench (0.5 g/tree) & $6.93 a^{D}$ & $7.04 a_{a} C D$ & $7.25 a_{a}^{C D}$ & $7.33 a^{B C}$ & $7.52 a_{a} A B C$ & $7.57 a^{A B}$ & $7.85 \mathrm{a}^{\mathrm{A}}$ \\
\hline IMI injection (0.125 g/tree) & $7.10 a^{D}$ & $7.24 \mathrm{a}^{C D}$ & 7.31 a $C D$ & $7.44 a_{a}^{B C}$ & $7.57 \mathrm{a} A B C$ & $7.66 a_{a}^{A B}$ & $7.83 \mathrm{a}^{\mathrm{A}}$ \\
\hline IMI injection (0.25 g/tree) & $7.06 a^{A}$ & $7.15 a^{A}$ & $7.21 \mathrm{a}^{\mathrm{A}}$ & $7.17 \mathrm{a}^{\mathrm{A}}$ & $7.28 a^{A}$ & $7.36 \mathrm{ab}^{\mathrm{A}}$ & $7.28 c^{\mathrm{A}}$ \\
\hline INA spray (0.25 g/tree) & $6.95 a^{C}$ & 7.21 a $B C$ & $7.32 a^{A B}$ & $7.42 a^{A B}$ & $7.53 \mathrm{a}^{\mathrm{AB}}$ & $7.60 a^{A}$ & $7.79 \mathrm{a}^{\mathrm{A}}$ \\
\hline INA spray (0.5 g/tree) & $7.08 a^{B}$ & $7.19 a^{B}$ & $7.26 a_{a}^{A B}$ & $7.31 a^{A B}$ & $7.34_{a} A B$ & $7.41 a^{A}$ & $7.43 b c^{A}$ \\
\hline INA soil drench ( $0.25 \mathrm{~g} /$ tree $)$ & $7.17 \mathrm{a}^{\mathrm{D}}$ & 7.25 a $C D$ & $7.32 \mathrm{a}^{C D}$ & $7.43 a^{B C}$ & $7.55 a_{a}^{A B C}$ & $7.69 a_{a}^{A B}$ & $7.87 \mathrm{a}^{\mathrm{A}}$ \\
\hline INA soil drench ( $0.5 \mathrm{~g} /$ tree $)$ & $6.91 \mathrm{a}$ & $7.17 a_{a}^{C D}$ & $7.27 a_{a}^{C D}$ & $7.39 a^{B C}$ & $7.52 a^{A B C}$ & $7.65 a_{a}^{A B}$ & $7.81 \mathrm{a}^{\mathrm{A}}$ \\
\hline INA injection (0.125 g/tree) & $6.84 a^{E}$ & $7.12 \mathrm{a} D E$ & $7.29 a_{a}^{C D}$ & $7.42 a^{B C D}$ & $7.55 a_{a}^{A B C}$ & $7.67 a^{A B}$ & $7.86 a^{A}$ \\
\hline INA injection ( $0.25 \mathrm{~g} /$ tree $)$ & $7.10 a^{A}$ & $7.21 \mathrm{a}^{\mathrm{A}}$ & $7.17 a^{A B}$ & $7.29 \mathrm{a}^{\mathrm{AB}}$ & $7.33 \mathrm{a}^{\mathrm{AB}}$ & $7.37 \mathrm{ab}^{\mathrm{A}}$ & $7.34 \mathrm{bc}{ }^{\mathrm{A}}$ \\
\hline SA spray (0.25 g/tree) & $6.88 a^{D}$ & $7.01{ }_{a}^{C D}$ & $7.27 a^{B C}$ & $7.35 a_{a}^{B C}$ & $7.46 a_{a}^{A B}$ & $7.61 \mathrm{a}^{A B}$ & $7.81 \mathrm{a}^{\mathrm{A}}$ \\
\hline SA spray (0.5 g/tree) & $6.93 a^{B}$ & $7.13 \mathrm{a}^{\mathrm{B}}$ & $7.24 a^{A B}$ & $7.28 a_{a}^{A B}$ & $7.31 \mathrm{a}^{\mathrm{A}}$ & $7.37 \mathrm{ab}^{\mathrm{A}}$ & $7.48 \mathrm{bc}{ }^{\mathrm{A}}$ \\
\hline SA soil drench (0.25 g/tree) & $7.14 a^{D}$ & $7.21 \mathrm{a} C D$ & $7.32 a^{B C D}$ & $7.39 a^{B C}$ & $7.56{ }_{a}^{A B C}$ & $7.69 a_{a}^{A B}$ & $7.86 \mathrm{a}^{\mathrm{A}}$ \\
\hline SA soil drench ( $0.5 \mathrm{~g} /$ tree $)$ & $6.85 \mathrm{a}^{\mathrm{D}}$ & 7.01 a $C D$ & $7.15 a_{a}^{C D}$ & $7.31 \mathrm{a}^{B C}$ & $7.55 a^{A B}$ & $7.66 a_{a}^{A B}$ & $7.82 \mathrm{a}^{\mathrm{A}}$ \\
\hline SA injection ( $0.125 \mathrm{~g} /$ tree $)$ & $7.07 \mathrm{a}^{\mathrm{D}}$ & $7.22 a_{a} C D$ & $7.33 a^{B C D}$ & $7.43 a^{B C}$ & $7.57 a_{a}^{A B}$ & $7.65 a^{A B}$ & $7.85 \mathrm{a}^{\mathrm{A}}$ \\
\hline SA injection ( $0.25 \mathrm{~g} /$ tree $)$ & $7.12 \mathrm{a}^{\mathrm{A}}$ & $7.24 a^{A}$ & $7.19 \mathrm{a}^{\mathrm{A}}$ & $7.21 \mathrm{a}^{\mathrm{A}}$ & $7.27 \mathrm{a}^{\mathrm{A}}$ & $7.22 b^{A}$ & $7.24 c^{\mathrm{A}}$ \\
\hline
\end{tabular}

${ }^{z}$ Data shown are the means of six replicates $(n=6)$. Values in the same row followed by different superscript uppercase letters are significantly different within each treatment $(P<0.05)$. Values in the same column followed by different subscript lowercase letters are significantly different at each sampling time $(P<0.05)$

\section{Discussion}

The present study evaluated the control effect of plant SAR inducers applied by foliar spray, soil drench, or trunk injection against citrus HLB under field conditions. The results indicated that trunk injection of the SAR inducers ASM, IMI, INA, or SA at $0.25 \mathrm{~g} /$ tree with three applications per year and foliar spray of INA or SA at $0.5 \mathrm{~g} /$ tree with six applications per year suppressed Las population growth within infected trees and reduced disease severity and pre-harvest fruit drop, and sustained fruit yield. Harnessing induced resistance for practical plant disease control represents a sustainable approach to crop protection (Durrant and Dong 2004). Therefore, the findings of this study may be useful for developing management programs with SAR inducers for effective control of citrus HLB. It is important to note that the present study was performed in a single grove of Hamlin sweet orange. Its performance in large-scale commercial citrus groves remains to be evaluated. More studies at multiple locations with various citrus cultivars or varieties under diverse climate conditions are required to adequately evaluate effects of such SAR inducer treatments for the control of citrus HLB.

Earlier studies have demonstrated that the efficacy of SAR inducers against citrus canker and HLB varied widely from limited to moderate under field conditions, depending on method, rate, frequency, and timing of application (Graham and Leite 2004; Graham and Myers 2011, 2013; Li et al. 2016, 2017; Hu et al. 2018). Results of this field trial appeared consistent with those earlier reports. When applied by foliar spray, two (INA and SA) of the four SAR inducers $(0.5 \mathrm{~g} /$ tree $)$ are effective for control of citrus HLB, but not at a lower rate $(0.25 \mathrm{~g} /$ tree). When applied by trunk injection, all the four SAR inducers $(0.25 \mathrm{~g} /$ tree $)$, but not at a lower rate $(0.125 \mathrm{~g} /$ tree), showed relatively fast and consistent effectiveness 
Table 2 Fruit yield of Hamlin sweet orange trees treated with various SAR inducers in a three-year field trial (2015-2018)

\begin{tabular}{|c|c|c|c|c|}
\hline \multirow[t]{2}{*}{ Treatment } & \multicolumn{4}{|c|}{ Yield (kg/tree) ${ }^{z}$} \\
\hline & 2015 & 2016 & 2017 & 2018 \\
\hline UTC & $50.7 \pm 5.9 a^{A}$ & $47.1 \pm 5.3 b^{A}$ & $41.5 \pm 5.8 a^{B}$ & $48.5 \pm 6.5 b^{\mathrm{A}}$ \\
\hline ASM spray (0.25 g/tree) & $52.1 \pm 5.2 \mathrm{a}^{\mathrm{A}}$ & $48.5 \pm 5.6 b_{b}^{A}$ & $40.4 \pm 7.2^{\mathrm{B}}$ & $48.9 \pm 6.2 b^{A}$ \\
\hline ASM spray (0.5 g/tree) & $51.4 \pm 6.7 a^{A}$ & $48.8 \pm 7.3 b^{A}$ & $42.5 \pm 6.1 a^{B}$ & $50.5 \pm 5.7 b^{A}$ \\
\hline ASM soil drench ( $0.25 \mathrm{~g} /$ tree $)$ & $49.5 \pm 5.3 \mathrm{a}^{\mathrm{A}}$ & $47.7 \pm 6.2 b^{A}$ & $40.8 \pm 5.9 a^{B}$ & $47.7 \pm 6.2 b^{A}$ \\
\hline ASM soil drench (0.5 g/tree) & $50.2 \pm 5.9 a^{A}$ & $47.5 \pm 5.7 b^{A}$ & $41.4 \pm 6.5 a^{B}$ & $49.1 \pm 6.6 b^{A}$ \\
\hline ASM injection (0.125 g/tree) & $51.1 \pm 5.5 \mathrm{a}^{\mathrm{A}}$ & $50.4 \pm 4.8 b^{A}$ & $39.7 \pm 5.6 a^{B}$ & $48.4 \pm 5.2 b^{A}$ \\
\hline ASM injection ( $0.25 \mathrm{~g} /$ tree $)$ & $49.8 \pm 6.4 a^{B}$ & $57.6 \pm 6.9 \mathrm{a}^{\mathrm{A}}$ & $43.5 \pm 5.3 \mathrm{a}^{\mathrm{C}}$ & $62.2 \pm 6.1 \mathrm{a}^{\mathrm{A}}$ \\
\hline IMI spray (0.25 g/tree) & $49.2 \pm 6.3 \mathrm{a}^{\mathrm{A}}$ & $48.3 \pm 6.1 b^{A}$ & $39.5 \pm 5.6_{a}^{B}$ & $50.4 \pm 5.8 b^{A}$ \\
\hline IMI spray (0.5 g/tree) & $52.7 \pm 5.1 \mathrm{a}^{\mathrm{A}}$ & $50.1 \pm 5.3 b^{A}$ & $42.6 \pm 6.4 a^{B}$ & $52.2 \pm 6.9 b^{\mathrm{A}}$ \\
\hline IMI soil drench ( $0.25 \mathrm{~g} /$ tree $)$ & $48.5 \pm 5.3 \mathrm{a}^{\mathrm{A}}$ & $46.7 \pm 6.0_{b}{ }^{A}$ & $40.4 \pm 5.5 a^{B}$ & $48.9 \pm 6.3 b^{A}$ \\
\hline IMI soil drench ( $0.5 \mathrm{~g} /$ tree $)$ & $50.6 \pm 5.9 a^{A}$ & $48.2 \pm 5.8 b^{A}$ & $39.9 \pm 6.2 \mathrm{a}^{\mathrm{B}}$ & $48.4 \pm 5.7 b^{\mathrm{A}}$ \\
\hline IMI injection (0.125 g/tree) & $47.7 \pm 6.6 a^{A}$ & $49.4 \pm 5.4_{b}{ }^{A}$ & $42.2 \pm 6.4_{a}^{B}$ & $49.6 \pm 6.1 b^{A}$ \\
\hline IMI injection ( $0.25 \mathrm{~g} /$ tree) & $51.9 \pm 6.1 a^{B}$ & $58.7 \pm 5.9 \mathrm{a}^{\mathrm{A}}$ & $43.6 \pm 6.5{ }_{a} C$ & $60.5 \pm 5.8 a^{A}$ \\
\hline INA spray (0.25 g/tree) & $49.5 \pm 6.3 \mathrm{a}^{\mathrm{A}}$ & $47.8 \pm 5.7 b^{A}$ & $39.1 \pm 5.2 \mathrm{a}^{\mathrm{B}}$ & $51.6 \pm 6.5 b^{A}$ \\
\hline INA spray ( $0.5 \mathrm{~g} /$ tree $)$ & $51.9 \pm 5.2 a^{B}$ & $52.7 \pm 6.5 \mathrm{ab}^{\mathrm{B}}$ & $41.2 \pm 5.9_{\mathrm{a}} \mathrm{C}$ & $60.7 \pm 6.3 a^{A}$ \\
\hline INA soil drench ( $0.25 \mathrm{~g} /$ tree $)$ & $48.6 \pm 6.1 \mathrm{a}^{\mathrm{A}}$ & $47.7 \pm 6.4 b^{A}$ & $39.3 \pm 4.5 \mathrm{a}^{\mathrm{B}}$ & $49.9 \pm 5.8 b^{A}$ \\
\hline INA soil drench (0.5 g/tree) & $50.3 \pm 5.9 a^{A}$ & $48.2 \pm 5.9 b^{A}$ & $40.6 \pm 5.2 \mathrm{a}^{\mathrm{B}}$ & $48.1 \pm 5.5 b^{A}$ \\
\hline INA injection (0.125 g/tree) & $49.2 \pm 5.5 \mathrm{a}^{\mathrm{A}}$ & $51.4 \pm 5.6 b^{A}$ & $43.2 \pm 6.9 \mathrm{a}^{\mathrm{B}}$ & $50.6 \pm 5.7 b^{A}$ \\
\hline INA injection (0.25 g/tree) & $51.1 \pm 6.4 a^{B}$ & $60.7 \pm 6.5 \mathrm{a}^{\mathrm{A}}$ & $43.2 \pm 6.9{ }_{a}{ }^{C}$ & $59.8 \pm 5.3 a^{A}$ \\
\hline SA spray (0.25 g/tree) & $50.5 \pm 7.1 \mathrm{a}^{\mathrm{A}}$ & $47.4 \pm 5.8 b_{b}^{A}$ & $39.3 \pm 5.1 a^{B}$ & $49.9 \pm 6.2 b^{A}$ \\
\hline SA spray ( $0.5 \mathrm{~g} /$ tree $)$ & $53.3 \pm 6.5 a^{B}$ & $54.6 \pm 6.7 \mathrm{ab}^{\mathrm{B}}$ & $43.4 \pm 4.6{ }_{a}{ }^{C}$ & $63.3 \pm 5.8 \mathrm{a}^{\mathrm{A}}$ \\
\hline SA soil drench ( $0.25 \mathrm{~g} /$ tree $)$ & $51.6 \pm 6.4 \mathrm{a}^{\mathrm{A}}$ & $48.7 \pm 6.3 b^{A}$ & $40.5 \pm 4.7_{a^{B}}$ & $50.9 \pm 5.4 b^{A}$ \\
\hline SA soil drench ( $0.5 \mathrm{~g} /$ tree $)$ & $50.2 \pm 5.5 \mathrm{a}^{\mathrm{A}}$ & $48.6 \pm 5.7 b^{A}$ & $40.8 \pm 5.6_{a}{ }^{B}$ & $51.2 \pm 6.3 b^{A}$ \\
\hline SA injection ( $0.125 \mathrm{~g} /$ tree $)$ & $48.6 \pm 6.2 \mathrm{a}^{\mathrm{A}}$ & $49.7 \pm 5.5 b^{A}$ & $42.3 \pm 5.9 \mathrm{a}^{\mathrm{B}}$ & $49.8 \pm 5.5 b^{A}$ \\
\hline SA injection (0.25 g/tree) & $51.5 \pm 5.6 a^{B}$ & $59.9 \pm 6.1 a^{A}$ & $43.9 \pm 7.7 a^{C}$ & $62.4 \pm 5.7 a^{A}$ \\
\hline 13-year old healthy tree ${ }^{y}$ & 112.6 & & & \\
\hline
\end{tabular}

${ }^{z}$ Data shown are the means \pm SD of six replicates $(n=6)$. Values in the same row followed by different superscript uppercase letters are significantly different within each treatment $(P<0.05)$. Values in the same column followed by different subscript lowercase letters are significantly different at each sampling time $(P<$ 0.05). ${ }^{y}$ averaged fruit yield of 13-year old, healthy Hamlin sweet orange trees in Florida reported by Roka et al. (2000) prior to the era of HLB

of disease control (Figs. 1, 2 and Tables 1, 2 and 3). In contrast, soil applications of the SAR inducers did not have significant control effect against HLB. Multiple factors might be responsible for such a varying efficacy. Among others, foliar spray and soil drench may be a significant limiting factor that results in poor intake of SAR inducers by a citrus plant. Many SAR inducers such as ASM and SA are highly photodegradable and biodegradable (Silva et al. 2007; Sleiman et al. 2017) and vulnerable to other environmental conditions including washoff by rainfall, whereas soil particles might absorb SAR inducers that reduces the uptake by roots. Trunk injection is a target-precise, efficient method for delivery of compounds into plants, and, may avoid problems of degradation and poor uptake of SAR inducers associated with foliar spray and soil drench (Aćimović et al. 2015). However, current injection technologies and equipment are labor intensive and costly, and consequently, may not be practical for large-scale applications in HLB endemic areas, such as Florida. Therefore, development of automated injection systems with high efficiency and low cost is needed for commercial citrus production. Meanwhile, how to improve the uptake efficacy of SAR inducers via foliar spray needs further investigation.

SAR inducers might suppress Las growth via two different mechanisms (Table 1). First, it may induce the production of antimicrobials. Earlier studies have demonstrated that a high and persistent upregulation of expression of $P R$ genes $P R 1,2,3$ in citrus in response to ASM, IMI, INA and SA (Dekkers et al. 2004; Francis et al. 2009; Li et al. 2017; $\mathrm{Hu}$ et al. 2018). The PR proteins PR1, PR2 and PR3 have antimicrobial activities against various plant pathogenic fungi, bacteria and oomycetes (Mauch et al. 1988; Alexander et al. 1993; 
Table 3 Pre-harvest fruit drop of Hamlin sweet orange trees treated with various SAR inducers in a three-year field trial (2015-2018)

\begin{tabular}{|c|c|c|c|c|}
\hline \multirow[t]{2}{*}{ Treatment } & \multicolumn{4}{|c|}{ No. of fruit dropped ${ }^{z}$} \\
\hline & 2015 & 2016 & 2017 & 2018 \\
\hline UTC & $136 \pm 29 a^{B}$ & $149 \pm 32 a^{B}$ & $214 \pm 43 a^{A}$ & $148 \pm 35_{a^{B}}$ \\
\hline ASM spray (0.25 g/tree) & $128 \pm 26 a^{B}$ & $139 \pm 28 a^{B}$ & $217 \pm 47 a^{A}$ & $137 \pm 33 a^{B}$ \\
\hline ASM spray (0.5 g/tree) & $139 \pm 32 a^{B}$ & $145 \pm 31 a^{B}$ & $229 \pm 45 a^{A}$ & $147 \pm 32 a^{B}$ \\
\hline ASM soil drench ( $0.25 \mathrm{~g} /$ tree $)$ & $133 \pm 27 a^{B}$ & $148 \pm 34 a^{B}$ & $237 \pm 46 a^{A}$ & $146 \pm 29 a^{B}$ \\
\hline ASM soil drench (0.5 g/tree) & $129 \pm 24_{a}^{B}$ & $141 \pm 28 a^{B}$ & $221 \pm 4 a_{a}{ }^{A}$ & $135 \pm 31 a^{B}$ \\
\hline ASM injection (0.125 g/tree) & $135 \pm 32 a^{B}$ & $124 \pm 30 a^{B}$ & $216 \pm 38 a^{A}$ & $129 \pm 33 a^{B}$ \\
\hline ASM injection ( $0.25 \mathrm{~g} /$ tree $)$ & $143 \pm 28 a^{B}$ & $78 \pm 21 b^{c}$ & $235 \pm 45 a^{A}$ & $69 \pm 24_{b}{ }^{c}$ \\
\hline IMI spray (0.25 g/tree) & $126 \pm 29 a^{B}$ & $139 \pm 33 a^{B}$ & $219 \pm 36 a^{A}$ & $144 \pm 30 a^{B}$ \\
\hline IMI spray (0.5 g/tree) & $138 \pm 31 \mathrm{a}^{\mathrm{B}}$ & $124 \pm 28 a^{B}$ & $228 \pm 41_{a}{ }^{A}$ & $152 \pm 37 a^{B}$ \\
\hline IMI soil drench ( $0.25 \mathrm{~g} /$ tree $)$ & $143 \pm 30 a^{B}$ & $132 \pm 31 a^{B}$ & $242 \pm 48 a^{A}$ & $137 \pm 31_{a}^{B}$ \\
\hline IMI soil drench ( $0.5 \mathrm{~g} /$ tree $)$ & $132 \pm 28 a^{B}$ & $147 \pm 35 a^{B}$ & $233 \pm 44 a^{A}$ & $144 \pm 33 a^{B}$ \\
\hline IMI injection (0.125 g/tree) & $145 \pm 34_{a}^{B}$ & $131 \pm 29 a^{B}$ & $226 \pm 39 a^{A}$ & $150 \pm 38 a^{B}$ \\
\hline IMI injection ( $0.25 \mathrm{~g} /$ tree) & $128 \pm 29 a^{B}$ & $71 \pm 20_{b}{ }^{c}$ & $215 \pm 37 a^{A}$ & $79 \pm 21 b^{c}$ \\
\hline INA spray (0.25 g/tree) & $121 \pm 29 a^{B}$ & $138 \pm 32 a^{B}$ & $237 \pm 48 a^{A}$ & $139 \pm 35 a^{B}$ \\
\hline INA spray ( $0.5 \mathrm{~g} /$ tree $)$ & $137 \pm 30 a^{B}$ & $145 \pm 35 a^{B}$ & $219 \pm 46_{a}{ }^{A}$ & $70 \pm 25 b^{B}$ \\
\hline INA soil drench ( $0.25 \mathrm{~g} /$ tree $)$ & $129 \pm 28 a^{B}$ & $139 \pm 32 a^{B}$ & $240 \pm 48 a^{A}$ & $142 \pm 31 a^{B}$ \\
\hline INA soil drench (0.5 g/tree) & $140 \pm 29 a^{B}$ & $151 \pm 31 a^{B}$ & $226 \pm 44 a^{A}$ & $133 \pm 30 a^{B}$ \\
\hline INA injection (0.125 g/tree) & $132 \pm 31_{a}^{B}$ & $127 \pm 27 a^{B}$ & $215 \pm 35 a^{A}$ & $127 \pm 34 a^{B}$ \\
\hline INA injection (0.25 g/tree) & $145 \pm 32 a^{B}$ & $72 \pm 20_{b}{ }^{c}$ & $230 \pm 43 a^{A}$ & $77 \pm 24_{b}{ }^{c}$ \\
\hline SA spray ( $0.25 \mathrm{~g} /$ tree $)$ & $136 \pm 27 \mathrm{a}^{\mathrm{B}}$ & $148 \pm 32 a^{B}$ & $242 \pm 49 a^{A}$ & $129 \pm 35 a^{B}$ \\
\hline SA spray (0.5 g/tree) & $129 \pm 30 a^{B}$ & $140 \pm 34 a^{B}$ & $227 \pm 44_{a}{ }^{A}$ & $81 \pm 22 b c$ \\
\hline SA soil drench ( $0.25 \mathrm{~g} /$ tree $)$ & $133 \pm 27 a^{B}$ & $148 \pm 34_{a}{ }^{B}$ & $237 \pm 46 a^{A}$ & $146 \pm 29 a^{B}$ \\
\hline SA soil drench $(0.5 \mathrm{~g} /$ tree $)$ & $142 \pm 32 a^{B}$ & $133 \pm 27 a^{B}$ & $222 \pm 48 a^{A}$ & $133 \pm 33 a^{B}$ \\
\hline SA injection ( $0.125 \mathrm{~g} /$ tree $)$ & $126 \pm 28 a^{B}$ & $140 \pm 31 a^{B}$ & $235 \pm 44_{a}{ }^{A}$ & $142 \pm 35 a^{B}$ \\
\hline SA injection ( $0.25 \mathrm{~g} /$ tree $)$ & $141 \pm 31_{a}^{B}$ & $77 \pm 23 b^{c}$ & $225 \pm 42 a^{A}$ & $69 \pm 21 b^{c}$ \\
\hline
\end{tabular}

${ }^{z}$ Data shown are the means \pm SD of six replicates $(n=6)$. Values in the same row followed by different superscript uppercase letters are significantly different within each treatment $(P<0.05)$. Values in the same column followed by different subscript lowercase letters are significantly different at each sampling time $(P<0.05)$

Rauscher et al. 1999; Maxson-Stein et al. 2002; Walters and Fountaine 2009). Furthermore, ASM spray-induced PR1 expression in tomato was positively associated with field control efficacy of Xanthomonas leaf spot and Pseudomonas bacterial speck tomato (Herman et al. 2007). Upregulation of PR2 expression was correlated with reduction of canker lesions on citrus leaves (Francis et al. 2009). These earlier reports suggested that the PR proteins might have antibacterial activity against bacterial pathogens including Las. Second, SAR inducers themselves might have direct antimicrobial activities against Las under certain conditions. For example, SA has antibacterial activity against Agrobacterium tumefaciens (Yuan et al. 2007; Anand et al. 2008) and Rhizobium meliloti (Martínez-Abarca et al. 1998) at relatively lower concentrations ( 5 to $25 \mu \mathrm{M}=0.69$ to $3.45 \mu \mathrm{g} / \mathrm{mL}$ ) in vitro. Las is a closely relative of Agrobacterium and Rhizobium, belonging to the family Rhizobiaceae (Duan et al. 2009). It is possible that application of the SAR inducers, and the consequent accumulation of SA in a citrus tree may have inhibitory effect on Las. Las encodes a functional SA hydroxylase that degrades SA, thus affecting its efficacy in inducing SAR in Las-infected citrus plants (Li et al. 2017). Trunk injection or multiple foliar sprays of SA at a relatively higher rate seems to be able to overcome the SA degradation by the enzyme.

In this study, all SAR inducer treatments did not result in significant reduction of Las titers in treated trees when compared year by year and the Las titer was consistently maintained in a relative high level of $10^{7}$ cells/g of leaf tissue throughout the experiments (Table 1). The results of this study were consistent with those in earlier studies that SAR inducer treatments had limited effect in reduction of the titers of bacterial pathogens within infected plants (Aćimović et al. 2015; Li et al. 2016; Hu et al. 2018). The antimicrobials induced by SAR inducers 
Table 4 Quality of fruit harvested from Hamlin sweet orange trees treated with SAR inducers in a three-year field trial (2015-2018) ${ }^{z}$

\begin{tabular}{|c|c|c|c|c|c|c|c|c|c|c|c|c|c|c|c|c|}
\hline \multirow[t]{2}{*}{ Treatment } & \multicolumn{4}{|c|}{ Percent juice content } & \multicolumn{4}{|l|}{ Brix } & \multicolumn{4}{|c|}{ Acidity $(\% \mathrm{w} / \mathrm{w})$} & \multicolumn{4}{|c|}{ Brix acidity ratio } \\
\hline & 2015 & 2016 & 2017 & 2018 & 015 & 016 & 017 & 2018 & 2015 & 2016 & 2017 & 2018 & 2015 & 2016 & 2017 & 2018 \\
\hline JTC & & $8 \mathrm{a}^{\mathrm{A}}$ & $54.0 a^{\mathrm{A}}$ & $55.7 \mathrm{a}^{\mathrm{A}}$ & $2 \mathrm{a}^{\mathrm{A}}$ & $7{ }^{\mathrm{A}}$ & $4 \mathrm{a}^{\mathrm{A}}$ & $1.8 a^{\mathrm{A}}$ & $0.64{ }_{a}^{A}$ & $0.63 \mathrm{a}^{\mathrm{A}}$ & $0.66{ }_{a}^{A}$ & $0.68 a^{A}$ & $17.6 a^{A}$ & $18.2_{\mathrm{a}}^{\mathrm{A}}$ & $17.4{ }_{a}^{\mathrm{A}}$ & $17.3^{A}$ \\
\hline$M$ & $6 \mathrm{a}^{\mathrm{A}}$ & $6 a^{A}$ & $53.9 \mathrm{a}^{\mathrm{A}}$ & $54.4 \mathrm{a}^{\mathrm{A}}$ & $.3 \mathrm{a}^{\mathrm{A}}$ & g A & $2 \mathrm{a}^{\mathrm{A}}$ & $.5 \mathrm{a}^{\mathrm{A}}$ & $65 a^{A}$ & $0.69 a^{A}$ & 0 & $67 a^{A}$ & $\mathrm{~A}^{\mathrm{A}}$ & $17.9_{\mathrm{a}}^{\mathrm{A}}$ & $18.3 \mathrm{a}^{\mathrm{A}}$ & 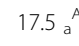 \\
\hline 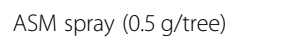 & $8 \mathrm{a}^{\mathrm{A}}$ & $7 \mathrm{a}^{\mathrm{A}}$ & $55.4 \mathrm{a}^{\mathrm{A}}$ & $54.8 \mathrm{a}^{\mathrm{A}}$ & $8 \mathrm{a}^{\mathrm{A}}$ & 1 & $1.4 \mathrm{a}^{\mathrm{A}}$ & $.7 \mathrm{a}^{\mathrm{A}}$ & $68 a^{A}$ & $61{ }_{a}^{A}$ & 0.64 & $0.65 \mathrm{a}^{\mathrm{A}}$ & $5 a^{A}$ & $18.3_{\mathrm{a}}^{\mathrm{A}}$ & $17.8 a^{A}$ & 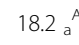 \\
\hline drench $(0.25$ & $55.3 \mathrm{a}^{\mathrm{A}}$ & $2 \mathrm{a}^{\mathrm{A}}$ & $53.9 \mathrm{a}^{\mathrm{A}}$ & $54.4 \mathrm{a}^{\mathrm{A}}$ & 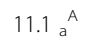 & $2.0 \mathrm{a}^{\mathrm{A}}$ & $a^{A}$ & ${ }^{A}$ & A & A & 0 & $0.68 a^{A}$ & $a^{A}$ & $\mathrm{a}^{\mathrm{A}}$ & ${ }^{A}$ & 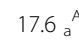 \\
\hline M soil drench (0.5 g/tree) & $54.2 \mathrm{a}^{\mathrm{A}}$ & $1 \mathrm{a}^{\mathrm{A}}$ & $53.2 \mathrm{a}^{\mathrm{A}}$ & $55.0 a^{A}$ & $.0 \mathrm{a}^{\mathrm{A}}$ & $7 a^{A}$ & $1.4 \mathrm{a}^{\mathrm{A}}$ & $.9 \mathrm{a}^{\mathrm{A}}$ & $62 a^{A}$ & $0.68 a^{A}$ & $0.64{ }_{a}^{A}$ & $0.67 \mathrm{a}^{\mathrm{A}}$ & $6{ }_{a}^{A}$ & $17.3_{a}^{A}$ & $17.7 \mathrm{a}^{\mathrm{A}}$ & $18.2 a^{A}$ \\
\hline M injection (0.125 g/tree) & $54.6 \mathrm{a}^{\mathrm{A}}$ & $52.8 \mathrm{a}^{\mathrm{A}}$ & $54.9 \mathrm{a}^{\mathrm{A}}$ & $53.4 \mathrm{a}^{\mathrm{A}}$ & $12.1 \mathrm{a}^{\mathrm{A}}$ & $11.3 \mathrm{a}^{\mathrm{A}}$ & $1.7 \mathrm{a}^{\mathrm{A}}$ & $1.8 \mathrm{a}^{\mathrm{A}}$ & $0.68{ }_{a}^{A}$ & $0.62 a^{A}$ & $0.67{ }_{a}^{A}$ & $0.65 \mathrm{a}^{\mathrm{A}}$ & $3.5{ }^{\mathrm{A}}$ & $17.7 \mathrm{a}^{\mathrm{A}}$ & $18.1 \mathrm{a}^{\mathrm{A}}$ & $18.3^{A}$ \\
\hline M injection $(0.25 \mathrm{~g} / \mathrm{t}$ & $55.1 \mathrm{a}^{\mathrm{A}}$ & $53.6 a^{\mathrm{A}}$ & $54.4 \mathrm{a}^{\mathrm{A}}$ & $55.2 \mathrm{a}^{\mathrm{A}}$ & $11.8 \mathrm{a}^{\mathrm{A}}$ & $11.4 \mathrm{a}^{\mathrm{A}}$ & $11.6 a^{A}$ & $1.7 \mathrm{a}^{\mathrm{A}}$ & $0.67{ }_{a}^{A}$ & $0.60 a^{A}$ & $0.62{ }^{A}{ }^{A}$ & $0.63 \mathrm{a}^{\mathrm{A}}$ & $17.9 \mathrm{a}^{\mathrm{A}}$ & $18.9 a^{\mathrm{A}}$ & $18.7{ }_{a}^{A}$ & $18.6 a^{A}$ \\
\hline $\mathrm{Ml} \mathrm{s}$ & $53.4 \mathrm{a}^{\mathrm{A}}$ & $54.6 \mathrm{a}^{\mathrm{A}}$ & $55.0 \mathrm{a}^{\mathrm{A}}$ & $54.5 \mathrm{a}^{\mathrm{A}}$ & $11.4 \mathrm{a}^{\mathrm{A}}$ & $11.6{ }^{\mathrm{A}}$ & $11.1 \mathrm{a}^{\mathrm{A}}$ & $11.6 \mathrm{a}^{\mathrm{A}}$ & $0.66{ }_{a}^{A}$ & $0.68 a^{A}$ & $0.65{ }^{\mathrm{A}}$ & $0.67{ }_{a}^{A}$ & $17.4{ }_{a}^{A}$ & $17.9 a^{\mathrm{A}}$ & $17.2 \mathrm{a}^{\mathrm{A}}$ & $17.7 \mathrm{a}^{\mathrm{A}}$ \\
\hline IMI spray (0.5 g/tree) & $53.9 \mathrm{a}^{\mathrm{A}}$ & $55.1 \mathrm{a}^{\mathrm{A}}$ & $54.6 a^{\mathrm{A}}$ & $53.8 \mathrm{a}^{\mathrm{A}}$ & $11.5 \mathrm{a}^{\mathrm{A}}$ & $11.1 \mathrm{a}^{\mathrm{A}}$ & $1.6{ }_{a}{ }^{\mathrm{A}}$ & $1.2 \mathrm{a}^{\mathrm{A}}$ & $0.64{ }_{a}^{A}$ & $0.63 \mathrm{a}^{\mathrm{A}}$ & $0.67{ }_{a}^{A}$ & $0.65 \mathrm{a}^{\mathrm{A}}$ & $17.9 \mathrm{a}^{\mathrm{A}}$ & $17.3^{\mathrm{A}}$ & $17.4{ }_{a}^{A}$ & $17.2 \mathrm{a}^{\mathrm{A}}$ \\
\hline ? & $55.1 \mathrm{a}^{\mathrm{A}}$ & $53.7 \mathrm{a}^{\mathrm{A}}$ & $54.4 \mathrm{a}^{\mathrm{A}}$ & $55.0 \mathrm{a}^{\mathrm{A}}$ & $12.0 \mathrm{a}^{\mathrm{A}}$ & $11.5 \mathrm{a}^{\mathrm{A}}$ & $4 \mathrm{a}^{\mathrm{A}}$ & ${ }^{{ }^{A}}$ & $0.69{ }_{a}^{A}$ & $0.66 a^{A}$ & $0.64{ }_{a}^{A}$ & $0.67 \mathrm{a}^{\mathrm{A}}$ & $17.4 \mathrm{a}^{\mathrm{A}}$ & $17.8_{a}^{A}$ & $17.7{ }^{\mathrm{A}}$ & 17 \\
\hline Ml & $n^{2}$ & $8 a^{\mathrm{A}}$ & $53.5 \mathrm{a}^{\mathrm{A}}$ & $54.1 \mathrm{a}^{\mathrm{A}}$ & $11.4 a^{\mathrm{A}}$ & 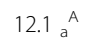 & $a^{A}$ & ${ }^{A}$ & $64 a^{A}$ & $0.69 a^{A}$ & $0.65 \mathrm{a}^{\mathrm{A}}$ & $0.68 a^{A}$ & ${ }_{a}^{A}$ & A & $\mathrm{a}^{\mathrm{A}}$ & $17.2_{\mathrm{a}}^{\mathrm{A}}$ \\
\hline Ml in & $53.2 \mathrm{a}^{\mathrm{A}}$ & A & $53.5 \mathrm{a}^{\mathrm{A}}$ & 5 & $11.6 \mathrm{a}^{\mathrm{A}}$ & 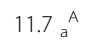 & A & A & A & 0.6 & $0.64{ }_{a}^{A}$ & ${ }^{A}$ & ${ }^{A}$ & $17.2^{\mathrm{A}}$ & 17 & $17.7_{a}^{A}$ \\
\hline ee) & $54.9 \mathrm{a}^{\mathrm{A}}$ & $53.8 \mathrm{a}^{\mathrm{A}}$ & $54.2 \mathrm{a}^{\mathrm{A}}$ & $55.1 \mathrm{a}^{\mathrm{A}}$ & $11.4 \mathrm{a}^{\mathrm{A}}$ & $11.3 \mathrm{a}^{\mathrm{A}}$ & $11.5 \mathrm{a}^{\mathrm{A}}$ & $11.4 \mathrm{a}^{\mathrm{A}}$ & $0.66{ }_{a}^{A}$ & $0.60 a^{A}$ & $0.62 \mathrm{a}^{\mathrm{A}}$ & $0.62 a^{A}$ & $17.3 \mathrm{a}^{\mathrm{A}}$ & $18.7 \mathrm{a}^{\mathrm{A}}$ & $18.5 \mathrm{a}^{\mathrm{A}}$ & $18.3_{a}^{A}$ \\
\hline NA Sp & $55.0 \mathrm{a}^{\mathrm{A}}$ & $53.3 \mathrm{a}^{\mathrm{A}}$ & $54.6 \mathrm{a}^{\mathrm{A}}$ & $53.2 \mathrm{a}^{\mathrm{A}}$ & $11.2 \mathrm{a}^{\mathrm{A}}$ & $11.5 \mathrm{a}^{\mathrm{A}}$ & $11.6 \mathrm{a}^{\mathrm{A}}$ & $11.3 \mathrm{a}^{\mathrm{A}}$ & $0.65{ }_{a}^{A}$ & $0.66 a^{A}$ & $0.68{ }_{a}^{A}$ & $0.64 \mathrm{a}^{\mathrm{A}}$ & $17.2 \mathrm{a}^{\mathrm{A}}$ & $17.4_{\mathrm{a}}^{\mathrm{A}}$ & $17.2 \mathrm{a}^{\mathrm{A}}$ & $17.6_{a}{ }^{A}$ \\
\hline A & $53.5 \mathrm{a}^{\mathrm{A}}$ & $52.4 \mathrm{a}^{\mathrm{A}}$ & $54.9 \mathrm{a}^{\mathrm{A}}$ & $53.5 \mathrm{a}^{\mathrm{A}}$ & $11.9 \mathrm{a}^{\mathrm{A}}$ & $11.1 \mathrm{a}^{\mathrm{A}}$ & $11.4 \mathrm{a}^{\mathrm{A}}$ & $11.7 \mathrm{a}^{\mathrm{A}}$ & $0.68{ }_{a}{ }^{A}$ & $0.63 \mathrm{a}^{\mathrm{A}}$ & $0.66{ }_{a}^{A}$ & $0.65 \mathrm{a}^{\mathrm{A}}$ & $17.5 \mathrm{a}^{\mathrm{A}}$ & $17.7_{\mathrm{a}}^{\mathrm{A}}$ & $17.3 \mathrm{a}^{\mathrm{A}}$ & $18.0_{a}^{A}$ \\
\hline NA soildrench $(025$ & $54.4 a^{\mathrm{A}}$ & $55.1 \mathrm{a}^{\mathrm{A}}$ & $53.6 a^{A}$ & $54.7 a^{A}$ & $11.6 a^{\mathrm{A}}$ & $11.7 \mathrm{a}^{\mathrm{A}}$ & $11.2 \mathrm{a}^{\mathrm{A}}$ & $11.5 \mathrm{a}^{\mathrm{A}}$ & $0.67{ }_{a}^{A}$ & $0.68 a^{A}$ & $0.63 \mathrm{a}^{\mathrm{A}}$ & $0.66 a^{A}$ & $17.3 \mathrm{a}^{\mathrm{A}}$ & $17.1_{\mathrm{a}}^{\mathrm{A}}$ & $17.8 a^{A}$ & $17.4_{a}^{A}$ \\
\hline 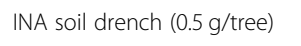 & $54.1 \mathrm{a}^{\mathrm{A}}$ & $53.7 \mathrm{a}^{\mathrm{A}}$ & $54.5 \mathrm{a}^{\mathrm{A}}$ & $53.8 \mathrm{a}^{\mathrm{A}}$ & $11.8 \mathrm{a}^{\mathrm{A}}$ & $11.1 \mathrm{a}^{\mathrm{A}}$ & $12.0 a^{A}$ & $11.4 \mathrm{a}^{\mathrm{A}}$ & $0.69{ }_{a}^{A}$ & $0.63 \mathrm{a}^{\mathrm{A}}$ & $0.69{ }_{a}^{A}$ & $0.64 \mathrm{a}^{\mathrm{A}}$ & $17.1 \mathrm{a}^{\mathrm{A}}$ & $17.6_{a}^{A}$ & $17.4{ }_{a}^{A}$ & $17.8^{A}$ \\
\hline 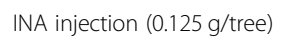 & $3.8 \mathrm{a}^{\mathrm{A}}$ & $54.8 \mathrm{a}^{\mathrm{A}}$ & $53.2 \mathrm{a}^{\mathrm{A}}$ & $54.3 \mathrm{a}^{\mathrm{A}}$ & $11.2 \mathrm{a}^{\mathrm{A}}$ & $12.1 \mathrm{a}^{\mathrm{A}}$ & $11.3 \mathrm{a}^{\mathrm{A}}$ & $11.7 \mathrm{a}^{\mathrm{A}}$ & $0.64{ }_{a}^{A}$ & $0.68 a^{A}$ & $0.64{ }_{a}^{A}$ & $0.66 a^{A}$ & $17.5 \mathrm{a}^{\mathrm{A}}$ & $17.8_{a}^{A}$ & $17.6^{\mathrm{A}}$ & $17.7_{\mathrm{a}}^{\mathrm{A}}$ \\
\hline NA in & $54.7 \mathrm{a}^{\mathrm{A}}$ & $53.9 \mathrm{a}^{\mathrm{A}}$ & $54.2 \mathrm{a}^{\mathrm{A}}$ & $55.0 \mathrm{a}^{\mathrm{A}}$ & $12.0 \mathrm{a}^{\mathrm{A}}$ & $11.6 a$ & $11.5 \mathrm{a}^{\mathrm{A}}$ & $11.8 \mathrm{a}^{\mathrm{A}}$ & $0.69{ }_{a}^{A}$ & $0.62 a^{A}$ & $0.61 \mathrm{a}^{\mathrm{A}}$ & $0.63 \mathrm{a}^{\mathrm{A}}$ & $17.4 \mathrm{a}^{\mathrm{A}}$ & $18.6{ }_{a}^{A}$ & $18.8 a^{\mathrm{A}}$ & $18.7_{a}^{A}$ \\
\hline A spray (0.25 g/tree) & $54.9 \mathrm{a}^{\mathrm{A}}$ & $53.5 \mathrm{a}^{\mathrm{A}}$ & $55.1 \mathrm{a}^{\mathrm{A}}$ & $54.2 \mathrm{a}^{\mathrm{A}}$ & $11.6 \mathrm{a}^{\mathrm{A}}$ & $12.1 \mathrm{a}^{\mathrm{A}}$ & $11.4 \mathrm{a}^{\mathrm{A}}$ & $11.5 \mathrm{a}^{\mathrm{A}}$ & $0.66{ }_{a}^{A}$ & $0.69 \mathrm{a}^{\mathrm{A}}$ & $0.64 \mathrm{a}^{\mathrm{A}}$ & $0.65 \mathrm{a}^{\mathrm{A}}$ & $17.6 \mathrm{a}^{\mathrm{A}}$ & $17.5_{a}^{A}$ & $17.8 \mathrm{a}^{\mathrm{A}}$ & $17.7_{a}^{A}$ \\
\hline (0.5 g/tree) & $53.8 \mathrm{a}^{\mathrm{A}}$ & $54.4 \mathrm{a}^{\mathrm{A}}$ & $54.2 \mathrm{a}^{\mathrm{A}}$ & $55.3 \mathrm{a}^{\mathrm{A}}$ & $11.9 \mathrm{a}^{\mathrm{A}}$ & $11.4 \mathrm{a}^{\mathrm{A}}$ & $10.9 \mathrm{a}^{\mathrm{A}}$ & $11.6 \mathrm{a}^{\mathrm{A}}$ & $0.68{ }_{a}^{A}$ & $0.65 \mathrm{a}^{\mathrm{A}}$ & $0.62{ }_{a}^{A}$ & $0.66 \mathrm{a}^{\mathrm{A}}$ & $17.5 \mathrm{a}^{\mathrm{A}}$ & $17.4_{a}^{A}$ & $17.6_{a}{ }^{A}$ & $17.5^{A}$ \\
\hline ee) & $54.2 \mathrm{a}^{\mathrm{A}}$ & $53.7 \mathrm{a}^{\mathrm{A}}$ & $54.6 a^{A}$ & $53.9 \mathrm{a}^{\mathrm{A}}$ & $10.9 \mathrm{a}^{\mathrm{A}}$ & $11.3 \mathrm{a}^{\mathrm{A}}$ & $11.5 \mathrm{a}^{\mathrm{A}}$ & $11.2 \mathrm{a}^{\mathrm{A}}$ & $0.63 \mathrm{a}^{\mathrm{A}}$ & $0.66 a^{A}$ & $0.64 \mathrm{a}^{\mathrm{A}}$ & $0.63 \mathrm{a}^{\mathrm{A}}$ & $17.3 \mathrm{a}^{\mathrm{A}}$ & $17.1 \mathrm{a}^{\mathrm{A}}$ & $18.0 a^{A}$ & $17.7_{a}^{A}$ \\
\hline A soil drench (0.5 g/tree) & $54.7 \mathrm{a}^{\mathrm{A}}$ & $55.1 \mathrm{a}^{\mathrm{A}}$ & $54.3 \mathrm{a}^{\mathrm{A}}$ & $53.9 \mathrm{a}^{\mathrm{A}}$ & $11.7 \mathrm{a}^{\mathrm{A}}$ & $11.3 \mathrm{a}^{\mathrm{A}}$ & $12.0 a^{A}$ & $11.5 \mathrm{a}^{\mathrm{A}}$ & $0.68 a^{A}$ & $0.64 \mathrm{a}^{\mathrm{A}}$ & $0.69 \mathrm{a}^{\mathrm{A}}$ & $0.66 a^{A}$ & $17.2 \mathrm{a}^{\mathrm{A}}$ & $17.7{ }_{a}^{A}$ & $17.4 \mathrm{a}^{\mathrm{A}}$ & $17.4 a^{A}$ \\
\hline SA injection ( $0.125 \mathrm{~g} /$ tree) & $53.5 \mathrm{a}^{\mathrm{A}}$ & $54.7 \mathrm{a}^{\mathrm{A}}$ & $55.2 \mathrm{a}^{\mathrm{A}}$ & $54.1 \mathrm{a}^{\mathrm{A}}$ & $11.6 \mathrm{a}^{\mathrm{A}}$ & $11.1 \mathrm{a}^{\mathrm{A}}$ & $11.5 \mathrm{a}^{\mathrm{A}}$ & $11.7 \mathrm{a}^{\mathrm{A}}$ & $0.66_{a}{ }^{A}$ & $0.63 \mathrm{a}^{\mathrm{A}}$ & $0.65{ }_{a}^{A}$ & $0.66{ }_{a}^{A}$ & $17.6{ }_{a}^{A}$ & $17.5_{a}^{A}$ & $17.7 \mathrm{a}^{\mathrm{A}}$ & $17.7 \mathrm{a}^{\mathrm{A}}$ \\
\hline A injection ( $0.25 \mathrm{~g} /$ tree) & 51 A & $53.8 \mathrm{a}^{\mathrm{A}}$ & $54.7 \mathrm{a}^{\mathrm{A}}$ & $11 \mathrm{~A}^{-} \mathrm{C}$ & $11.8 \mathrm{a}^{\mathrm{A}}$ & 113 & $11.4 \mathrm{a}^{\mathrm{A}}$ & $11.6 \mathrm{a}^{\mathrm{A}}$ & $0.68{ }_{a}^{A}$ & $0.61 \mathrm{a}^{\mathrm{A}}$ & $0.62 a^{A}$ & $0.63 \mathrm{a}^{\mathrm{A}}$ & $174^{\mathrm{A}} \mathrm{C}$ & $18.5_{a}^{A}$ & $18.4{ }_{a}^{A}$ & \\
\hline
\end{tabular}

${ }^{z}$ Data shown are the means of six replicates $(n=6)$. Values in the same row followed by same superscript uppercase letters are not significantly different within each treatment for each parameter of fruit quality $(P>0.05)$. Values in the same column followed by same subscript lowercase letters are not significantly different at each sampling time for different treatments $(P>0.05)$

in treated plants might not reach sufficiently high concentrations to kill or inhibit the growth of a large portion of bacterial cells in individual plants. Another potential explanation is that the HLB damages for the tested trees are too severe to respond to and recover from the treatments. Our previous study demonstrates that induction of plant defense by chemical inducers is more effective to young trees with mild HLB symptoms than older trees with severe HLB symptoms ( $\mathrm{Li}$ et al. 2016).

The significant reduction of pre-harvest fruit drop by trunk-injected SAR inducers at $0.25 \mathrm{~g} /$ tree (Table 3) might be attributed to their suppressive effects on Las population growth in HLB diseased citrus trees. Excessive pre-harvest fruit drop, usually happening three to 4 months prior to harvest, is positively correlated with HLB disease severity (Tang et al. 2019). Earlier work has speculated that altered balance among the plant hormones abscisic acid, auxin, ethylene, and jasmonic acid caused by Las infection played a critical role in increasing preharvest fruit drop in HLB disease trees (Martinelli et al.
2012; Nehela et al. 2018). Most recently, the oxidative stress induced by Las infection was suggested to result in cell wall modification, causing cell separation, and eventually promote pre-harvest fruit drop in infected plants (Tang and Vashisth 2020). Furthermore, it was proposed that the HLB-tolerant 'LB8-9' mandarin might have an advanced antioxidant system to mitigate the pathogeninduced oxidative stress, thereby contributing to its significantly decreased pre-harvest fruit drop relative to the HLB-susceptible Hamlin sweet orange (Tang and Vashisth 2020). Whether the SAR inducers tested in this study improve the antioxidant system to mitigate the Las infection-induced pre-harvest fruit drop needs to be investigated.

Previous studies show that some plant defense inducers applied to citrus are effective in improvement of fruit quality, with a higher juice content and/or higher brix acidity ratio ( $\mathrm{Li}$ et al. 2016; $\mathrm{Hu}$ et al. 2018). The results of this study indicate all the SAR inducers applied had trivial positive effect on fruit quality. ASM, IMI, INA, or SA injections at $0.25 \mathrm{~g} /$ tree resulted in a lower 


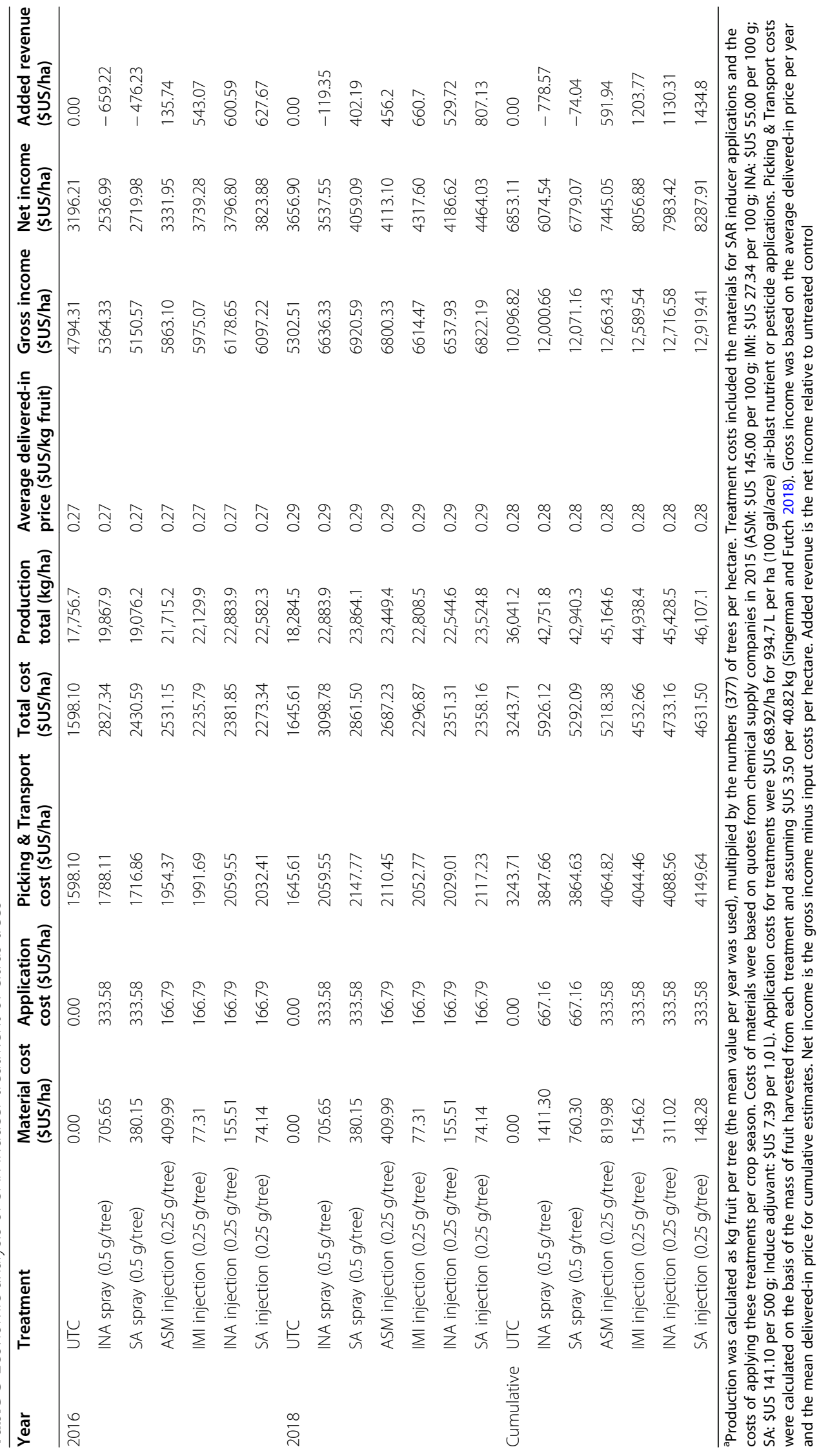


acidity and higher brix acidity ratio compared with the UTC or compared among years, but the differences were not statistically significant (Table 4). The discrepancy between this study and previous studies may be because of the differences in tree age, HLB disease severity, physiological conditions of the treated citrus trees, and the differences in local climate conditions. The spray treatment of INA or SA ( $0.5 \mathrm{~g} /$ tree) increased yield compared with the untreated control at the end of this trial, but its cost was over the revenue and might not be profitable in its present form (Table 5). Trunk injection of ASM, IMI, INA, or SA at $0.25 \mathrm{~g} /$ tree consistently increased yields compared with the untreated control in both 2016 and 2018, and the estimated revenue exceeded the total cost, with an assumed application cost equal to spray (Table 5). Therefore, these treatments might be profitable with the prerequisite that automated injection systems with high efficiency and low cost are available for commercial citrus production as discussed earlier.

\section{Conclusions}

This study showed that three trunk injections of the SAR inducers ASM, IMI, INA, or SA at a relatively low rate $(0.25 \mathrm{~g} /$ tree $)$ annually exhibited significant positive effects to slow down HLB disease progression and sustain fruit yield. Trunk injection of SAR inducers is more effective in controlling HLB than foliar spray and soil drench, and might be profitable. Further investigations are required to optimize injection systems for large-scale applications and to optimize SAR inducer uptake via foliar spray for HLB disease control. Finally, this study provides useful information regarding developing management programs with SAR inducers for effective control of citrus HLB.

\section{Methods}

\section{Field sites and experimental design}

The field trial was conducted with 17-year-old Hamlin sweet orange (Citrus sinensis [L.] Osbeck) on Swingle citrumelo (C. paradisi Macf. 'Duncan' grapefruit $\times$ Poncirus trifoliata [L.] Raf.) rootstock trees planted in 1999 in a citrus grove at Citrus Research and Education Center (CREC) in Lake Alfred, Florida from 2015 to 2018. The grove was established on the well-drained soil of Astatula fine sand (Typic Quartzipsamment) and with all trees being HLB positive. The grove received standard commercial care including normal irrigation and fertilization as well as conventional pest management practices throughout the experiments. One-week prior to the start of the trial, a field survey was carried out to determine the HLB severity via visual assessment and Las titers using quantitative real-time PCR (qPCR) assays. Trees with similar HLB severity were selected for the tests. Treatments were arranged in a randomized complete block design (RCBD) with 25 treatments (including an untreated control) replicated three times in blocks of two contiguous trees. Eight foliar spray treatments were ASM (Actigard 50 WG, 50\% a. i., Syngenta Crop Protection), IMI (Admire Pro, 42.8\% a.i.; Bayer Crop Science), INA (Astatech Inc., PA), and SA (SigmaAldrich, St. Louis, MO) at 0.25 and $0.5 \mathrm{~g} /$ tree per application that were applied six times per year. Eight soil drench treatments included ASM, IMI, INA, and SA at 0.25 and $0.5 \mathrm{~g} /$ tree per application in six applications per year. Eight trunk injection treatments consisted of ASM, IMI, INA, and SA at 0.125 and $0.25 \mathrm{~g} /$ tree per application in three applications per year. Products were mixed with water. The untreated control trees received a water-only spray treatment at each foliar spray time. The materials, application rates, and number and dates of application for each treatment were listed in Table 6.

\section{Treatment applications}

Six sprays per year were conducted by bi-monthly spraying $\sim 2.0 \mathrm{~L}$ of the treatment solution with $2.5 \mathrm{~mL} / \mathrm{L}$ of Induce non-ionic spreader-sticker adjuvant (Helena Chemical Company) onto each tree at sunny days. Treatments were initiated during the fall flush in early November 2015. Individual treatments were applied with a power-pumped sprayer at $400 \mathrm{kPa}$ of air pressure until runoff to ensure complete coverage.

Six soil applications per year were made bi-monthly as soil drenches at $4.0 \mathrm{~L} /$ tree to the soil surface in a crescent within 75 to $100 \mathrm{~cm}$ of the trunk on the top side of the bed to minimize runoff at sunny days during the spring, summer, summer-fall, and fall flush. Treatments were initiated during the fall flush in early November 2015. To facilitate infiltration, microsprinkler irrigation was applied for a few minutes to prewet the soil surface before drenching.

For trunk injection, one injection port per tree positioned at approximately $20 \mathrm{~cm}$ above bud union was made by drilling $20-30 \mathrm{~mm}$ into the xylem tissue with a $7.14 \mathrm{~mm}$ drill bit. The port was sealed with Arborplug ${ }^{\circ}$ No. 3 using Arborplug setter and a rubber hammer (Arborjet Inc., Woburn, MA, USA). Treatment solution in a pressurized bottle was injected into the trunk through the port using Arborjet tree I.V. at the recommended pressure $(\sim 345 \mathrm{kPa})$ during sunny days. The area surrounding drilling site was sprayed with $20 \mathrm{~mL}$ of Mefenoxam solution ( $337.5 \mathrm{ml} / \mathrm{L}$ RidomilGold ${ }^{\circ} \mathrm{SL}$, Syngenta Crop Protection) at the maximum label rate (2337 $\mathrm{mL}$ per hectare per application) for citrus based on 346 trees per hectare to prevent infection by Phytophthora spp. Each treatment required $12-24 \mathrm{~h}$ to inject the solution $(400 \mathrm{~mL})$ into the tree. Three injections per year of different treatments were conducted when new flush 
Table 6 Treatments applied to Hamlin sweet orange trees in a three-year field trial (2015-2018) at CREC-Lake Alfred, FL

\begin{tabular}{|c|c|c|c|c|c|c|}
\hline \multirow[t]{2}{*}{ Treatment $^{\mathrm{a}}$} & \multicolumn{3}{|c|}{ Applications } & \multicolumn{3}{|l|}{ Application dates } \\
\hline & Number ${ }^{b}$ & $\begin{array}{l}\text { Rate }^{c} \\
\text { (g/tree) }\end{array}$ & $\begin{array}{l}\begin{array}{l}\text { Volume } \\
\text { (mL/tree) }\end{array} \\
\end{array}$ & $2015 \& 2016$ & 2017 & 2018 \\
\hline UTC & 6 & - & 2000 & $11 / 2,2 / 2,4 / 5,6 / 3,8 / 5,10 / 10,12 / 8$ & $2 / 1,4 / 6,6 / 8,8 / 10,10 / 12,12 / 7$ & $2 / 5,4 / 3,6 / 7,8 / 9,10 / 12$ \\
\hline ASM spray & 6 & 0.25 & 2000 & $11 / 2,2 / 2,4 / 5,6 / 3,8 / 5,10 / 10,12 / 8$ & $2 / 1,4 / 6,6 / 8,8 / 10,10 / 12,12 / 7$ & $2 / 5,4 / 3,6 / 7,8 / 9,10 / 12$ \\
\hline ASM spray & 6 & 0.5 & 2000 & $11 / 2,2 / 2,4 / 5,6 / 3,8 / 5,10 / 10,12 / 8$ & $2 / 1,4 / 6,6 / 8,8 / 10,10 / 12,12 / 7$ & $2 / 5,4 / 3,6 / 7,8 / 9,10 / 12$ \\
\hline ASM soil drench & 6 & 0.25 & 4000 & $11 / 2,2 / 2,4 / 5,6 / 3,8 / 5,10 / 10,12 / 8$ & $2 / 1,4 / 6,6 / 8,8 / 10,10 / 12,12 / 7$ & $2 / 5,4 / 3,6 / 7,8 / 9,10 / 12$ \\
\hline ASM soil drench & 6 & 0.5 & 4000 & $11 / 2,2 / 2,4 / 5,6 / 3,8 / 5,10 / 10,12 / 8$ & $2 / 1,4 / 6,6 / 8,8 / 10,10 / 12,12 / 7$ & $2 / 5,4 / 3,6 / 7,8 / 9,10 / 12$ \\
\hline ASM trunk injection & 3 & 0.125 & 400 & $11 / 3,2 / 3,6 / 4,10 / 11$ & $2 / 2,6 / 9,10 / 13$ & $2 / 6,6 / 8,10 / 15$ \\
\hline ASM trunk injection & 3 & 0.25 & 400 & $11 / 3,2 / 3,6 / 4,10 / 11$ & $2 / 2,6 / 9,10 / 13$ & $2 / 6,6 / 8,10 / 15$ \\
\hline IMI spray & 6 & 0.25 & 2000 & $11 / 2,2 / 2,4 / 5,6 / 3,8 / 5,10 / 10,12 / 8$ & $2 / 1,4 / 6,6 / 8,8 / 10,10 / 12,12 / 7$ & $2 / 5,4 / 3,6 / 7,8 / 9,10 / 12$ \\
\hline IMI spray & 6 & 0.5 & 2000 & $11 / 2,2 / 2,4 / 5,6 / 3,8 / 5,10 / 10,12 / 8$ & $2 / 1,4 / 6,6 / 8,8 / 10,10 / 12,12 / 7$ & $2 / 5,4 / 3,6 / 7,8 / 9,10 / 12$ \\
\hline IMI soil drench & 6 & 0.25 & 4000 & $11 / 2,2 / 2,4 / 5,6 / 3,8 / 5,10 / 10,12 / 8$ & $2 / 1,4 / 6,6 / 8,8 / 10,10 / 12,12 / 7$ & $2 / 5,4 / 3,6 / 7,8 / 9,10 / 12$ \\
\hline IMI soil drench & 6 & 0.5 & 4000 & $11 / 2,2 / 2,4 / 5,6 / 3,8 / 5,10 / 10,12 / 8$ & $2 / 1,4 / 6,6 / 8,8 / 10,10 / 12,12 / 7$ & $2 / 5,4 / 3,6 / 7,8 / 9,10 / 12$ \\
\hline IMI trunk injection & 3 & 0.125 & 400 & $11 / 3,2 / 3,6 / 4,10 / 11$ & $2 / 2,6 / 9,10 / 13$ & $2 / 6,6 / 8,10 / 15$ \\
\hline IMI trunk injection & 3 & 0.25 & 400 & $11 / 3,2 / 3,6 / 4,10 / 11$ & $2 / 2,6 / 9,10 / 13$ & $2 / 6,6 / 8,10 / 15$ \\
\hline INA spray & 6 & 0.25 & 2000 & $11 / 2,2 / 2,4 / 5,6 / 3,8 / 5,10 / 10,12 / 8$ & $2 / 1,4 / 6,6 / 8,8 / 10,10 / 12,12 / 7$ & $2 / 5,4 / 3,6 / 7,8 / 9,10 / 12$ \\
\hline INA spray & 6 & 0.5 & 2000 & $11 / 2,2 / 2,4 / 5,6 / 3,8 / 5,10 / 10,12 / 8$ & $2 / 1,4 / 6,6 / 8,8 / 10,10 / 12,12 / 7$ & $2 / 5,4 / 3,6 / 7,8 / 9,10 / 12$ \\
\hline INA soil drench & 6 & 0.25 & 4000 & $11 / 2,2 / 2,4 / 5,6 / 3,8 / 5,10 / 10,12 / 8$ & $2 / 1,4 / 6,6 / 8,8 / 10,10 / 12,12 / 7$ & $2 / 5,4 / 3,6 / 7,8 / 9,10 / 12$ \\
\hline INA soil drench & 6 & 0.5 & 4000 & $11 / 2,2 / 2,4 / 5,6 / 3,8 / 5,10 / 10,12 / 8$ & $2 / 1,4 / 6,6 / 8,8 / 10,10 / 12,12 / 7$ & $2 / 5,4 / 3,6 / 7,8 / 9,10 / 12$ \\
\hline INA trunk injection & 3 & 0.125 & 400 & $11 / 3,2 / 3,6 / 4,10 / 11$ & $2 / 2,6 / 9,10 / 13$ & $2 / 6,6 / 8,10 / 15$ \\
\hline INA trunk injection & 3 & 0.25 & 400 & $11 / 3,2 / 3,6 / 4,10 / 11$ & $2 / 2,6 / 9,10 / 13$ & $2 / 6,6 / 8,10 / 15$ \\
\hline SA spray & 6 & 0.25 & 2000 & $11 / 2,2 / 2,4 / 5,6 / 3,8 / 5,10 / 10,12 / 8$ & $2 / 1,4 / 6,6 / 8,8 / 10,10 / 12,12 / 7$ & $2 / 5,4 / 3,6 / 7,8 / 9,10 / 12$ \\
\hline SA spray & 6 & 0.5 & 2000 & $11 / 2,2 / 2,4 / 5,6 / 3,8 / 5,10 / 10,12 / 8$ & $2 / 1,4 / 6,6 / 8,8 / 10,10 / 12,12 / 7$ & $2 / 5,4 / 3,6 / 7,8 / 9,10 / 12$ \\
\hline SA soil drench & 6 & 0.25 & 4000 & $11 / 2,2 / 2,4 / 5,6 / 3,8 / 5,10 / 10,12 / 8$ & $2 / 1,4 / 6,6 / 8,8 / 10,10 / 12,12 / 7$ & $2 / 5,4 / 3,6 / 7,8 / 9,10 / 12$ \\
\hline SA soil drench & 6 & 0.5 & 4000 & $11 / 2,2 / 2,4 / 5,6 / 3,8 / 5,10 / 10,12 / 8$ & $2 / 1,4 / 6,6 / 8,8 / 10,10 / 12,12 / 7$ & $2 / 5,4 / 3,6 / 7,8 / 9,10 / 12$ \\
\hline SA trunk injection & 3 & 0.125 & 400 & $11 / 3,2 / 3,6 / 4,10 / 11$ & $2 / 2,6 / 9,10 / 13$ & $2 / 6,6 / 8,10 / 15$ \\
\hline SA trunk injection & 3 & 0.25 & 400 & $11 / 3,2 / 3,6 / 4,10 / 11$ & $2 / 2,6 / 9,10 / 13$ & $2 / 6,6 / 8,10 / 15$ \\
\hline
\end{tabular}

a UTC: untreated control, ASM: acibenzolar-S-methyl, IMI: imidacloprid, INA: 2,6-dichloroisonicotinic acid, SA: salicylic acid

${ }^{\mathrm{b}}$ Number of applications per year

${ }^{c}$ Amount of treatment (a.i.) per tree per application

was present in spring, summer, and fall. Treatments were initiated during the fall flush in early November 2015.

\section{HLB disease severity assessment}

Disease severity data were collected by visual assessments as described by Gottwald et al. (2007) on a 6month interval from November 2015 through October 2018. Briefly, the canopy was divided horizontally and vertically into 8 sections and disease severity was scored for each section using a scale from 0 to 5 that indicates the proportion of limbs expressing HLB symptoms, where $0=$ no limbs; $1=1$ to $20 \%$ limbs; $2=21$ to $40 \%$ limbs; $3=41$ to $60 \%$ limbs; $4=61$ to $80 \%$ limbs; and $5=$ 81 to $100 \%$ limbs. An overall severity rating of 0 to 40 for each tree was obtained by summarizing these 8 scores of an individual tree and used to calculate the value of disease severity, expressed as the standardized area under the disease progress stair (sAUDPS) as described by Simko and Piepho (2012).

\section{Quantitative real-time PCR assays for evaluation of Las titers in citrus leaves}

To estimate the Las titer in treated trees, eight leaves with mottling symptoms were randomly collected from each tree to pool together as one biological replicate. For each pooled sample, $100 \mathrm{mg}$ of midribs was excised for DNA extraction. DNA was extracted from macerated midrib tissues using the Wizard Genomic DNA purification kit (Promega) following the manufacturer's recommendations. The air-dried DNA pellet was dissolved in $50-100 \mu \mathrm{L}$ of DNA rehydration solution (Promega) and 
kept at $-20^{\circ} \mathrm{C}$. Quantitative real-time PCR (qPCR) was performed with the Las specific primer-probe set "CQULA04F-CQULAP10-CQULA04R" (Wang et al. 2006; Trivedi et al. 2009) using an ABI PRISM 7500 sequence detection system (Applied Biosystems, Foster City, CA). qPCR was performed in $20 \mu \mathrm{L}$ of RT-qPCR reaction mixture that contained $10 \mu \mathrm{L}$ of $2 \times$ Quantitect probe PCR master mix (Qiagen, Valencia, CA), $500 \mathrm{nM}$ of each primer, $200 \mathrm{nM}$ of probe, and $100 \mathrm{ng}$ of DNA template. The qPCR reactions included $95^{\circ} \mathrm{C}$ denaturation for 15 min, followed by 40 cycles of $94^{\circ} \mathrm{C}$ for $15 \mathrm{~s}$ and $60^{\circ} \mathrm{C}$ for $1 \mathrm{~min}$. All DNA samples were run in triplicate. The cycle threshold $(\mathrm{Ct})$ values were obtained by adjusting threshold to the recommended level of 0.02 and were converted to the estimated bacterial titers with a standard equation as described by Li et al. (2016).

\section{Fruit drop, yield and quality analyses}

Pre-harvest fruit dropped was counted when fruit was harvested in each crop season. Fruits were harvested and weighed in December 2015, 2016, 2017, and 2018. The yield data were collected for each treated tree. For each treatment, a bag of fruit sample $(\sim 8.5 \mathrm{~kg})$ was randomly sampled from each of three selected trees and subjected to fruit quality analysis using a standard procedure (Gottwald et al. 2012). Juice content and fruit acidity were expressed as percent juice and percent citric acid. Total soluble solids were expressed as fruit brix (the measure of sugar content in fruit, i.e., $1 \mathrm{~g}$ of sugar $/ 100 \mathrm{~g}$ of juice is equivalent to $1^{\circ}$ of Brix). Thereafter, fruit brix and acidity ratio were calculated accordingly.

\section{Economic analysis}

The costs and benefits of treatments were estimated by multiplying yield ( $\mathrm{kg}$ fruit/ha) by the published values of marketable fruit per ha for the 2016 and 2018 growing seasons. The costs included the materials for SAR inducers and the costs of applying these treatments per growing season. Material costs were based on quotes from chemical supply companies in 2015 (ASM: \$US 145.00 per 100 g; IMI: \$US 27.34 per 100 g; INA: \$US 55.00 per 100 g; SA: \$US 141.10 per 500 g; Induce adjuvant: \$US 7.39 per $1.0 \mathrm{~L}$ ). Application costs for spray treatments were \$US 68.92/ha for $934.7 \mathrm{~L}$ per ha $(100$ gal/acre) air-blast nutrient or pesticide applications (Tansey et al. 2017). No published value for application cost of trunk injection was available at the time this work, so the application cost of trunk injection was estimated with that of spray. Costs also included transport and picking costs of harvested oranges (\$US 3.50 per $40.82 \mathrm{~kg}$ ) (Singerman and Futch 2018). Mean deliveredin prices for Hamlin oranges for processing in Florida were \$US $0.27 / \mathrm{kg}$ fruit (\$US 11.16 per $40.82 \mathrm{~kg}$ ) for 2016-2017 (National Agricultural Statistics Service
2018) and \$US $0.29 / \mathrm{kg}$ fruit (\$US 11.97 per $40.82 \mathrm{~kg}$ ) for 2018-2019 (National Agricultural Statistics Service 2020). Net income was calculated by subtracting total cost from the gross income per ha for each treatment. Added revenue was obtained by subtracting the net income of untreated control from the net income of each treatment.

\section{Statistical analysis}

All data analyses were performed using SAS V9.4 (SAS Institute Inc., Cary, NC). The data were first tested for normality and homogeneity of variance using the Shapiro-Wilk test and Levene's test, respectively. Prior to analysis, Las titers were $\log _{10}$ transformed to satisfy assumptions of normality and homoscedasticity. The main effects of treatment and time on Las titer, fruit drop, yield, and fruit quality were determined using repeated measures analysis of variance (ANOVA) with the MIXED procedure in SAS, with the first order autoregressive (AR (1)) covariance structure option based on the Akaikei and Bayesian information criteria. When treatment effects were significant $(P<0.05)$, mean separation was made by pairwise comparisons using the LSMEANS statement with the Tukey honestly significant difference $(\alpha=0.05)$ adjustment. When interactions of factors were significant $(P<0.05)$, the SLICE option of the LSMEANS statement was used to identify the significant factor effect. A one-way ANOVA was performed to determine any differences in treatment effects on sAUDPS and the treatment means were separated using Tukey's HSD test $(\alpha=0.05)$.

\section{Abbreviations}

ANOVA: Analysis of variance; ASM: Acibenzolar-S-methyl; BTH: Benzothiadiazole; CREC: Citrus Research and Education Center;

FL: Florida; HLB: Huanglongbing; IMI: Imidacloprid; INA: 2,6dichloroisonicotinic acid; Las: Candidatus Liberibacter asiaticus; Laf: Candidatus Liberibacter africanus; Lam: Candidatus Liberibacter americanus; PR: Pathogenesis-related; qPCR: Quantitative real-time PCR; SA: Salicylic acid; SAR: Systemic acquired resistance; SAUDPS: Standardized area under the disease progress stairs

\section{Acknowledgements}

The authors would like to thank the staff in the Grove Department at Citrus Research and Education Center in the University of Florida for their management of the experimental grove with fertilization, irrigation and application of pesticides.

\section{Authors' contributions}

NW and $J L$ conceived and designed the experiments. JL, VK, ZP, SD, DL, YH, $J X, D P, T L$, and NW carried out the experiments. $J L$ and NW analyzed the data and wrote the manuscript. All authors read and approved the final manuscript.

\section{Funding}

This work was supported by the Citrus Research and Development Foundation and Florida Department of Agriculture and Consumer Service Citrus Initiative Program.

Availability of data and materials Not applicable. 


\section{Ethics approval and consent to participate}

Not applicable.

\section{Consent for publication}

Not applicable.

\section{Competing interests}

The authors declare that they have no competing interests.

Received: 28 August 2020 Accepted: 27 November 2020

Published online: 11 January 2021

\section{References}

Aćimović SG, Meredith CL. Evaluation of newer biologicals and the SAR-activator candidate regalia in fire blight control applied by spraying or trunk injection. Fruit Quarterly. 2017;25(4):25-9.

Aćimović SG, Zeng Q, Mcghee GC, Sundin GW, Wise JC. Control of fire blight (Erwinia amylovora) on apple trees with trunk-injected plant resistance inducers and antibiotics and assessment of induction of pathogenesisrelated protein genes. Front Plant Sci. 2015;6:16.

Agostini JP, Bushong PM, Timmer LW. Greenhouse evaluation of products that induce host resistance for control of scab, melanose, and Alternaria brown spot of citrus. Plant Dis. 2003;87:69-74.

Akula N, Zheng H, Han FQ, Wang N. Discovery of novel SecA inhibitors of Candidatus Liberibacter asiaticus by structure based design. Bioorg Med Chem Lett. 2011;21:4183-8.

Alexander D, Goodman RM, Gut-Rella M, Glascock C, Weymann K, Friedrich L, et al. Increased tolerance to two oomycete pathogens in transgenic tobacco expressing pathogenesis-related protein 1a. Proc Natl Acad Sci U S A. 1993; 90:7327-31.

Anand A, Uppalapati SR, Ryu C-M, Allen SN, Kang L, Tang Y, et al. Salicylic acid and systemic acquired resistance play a role in attenuating crown gall disease caused by Agrobacterium tumefaciens. Plant Physiol. 2008;146:703-15.

Andrade M, Li J, Wang N. Candidatus Liberibacter asiaticus: virulence traits and control strategies. Trop Plant Pathol. 2020;45:285-97.

Andrade M, Pang Z, Achor DS, Wang H, Yao T, Singer BH, et al. The flagella of 'Candidatus Liberibacter asiaticus' and its movement in planta. Mol Plant Pathol. 2019;21:109-23.

Andrade M, Wang N. The tad pilus apparatus of Candidatus Liberibacter asiaticus and its regulation by VisNR. Mol Plant Microbe Interact. 2019;32:1175-87.

Aranega-Bou P, de la O'Leyva M, Finiti I, Garcia-Agustin P, Gonzalez-Bosch C. Priming of plant resistance by natural compounds. Hexanoic acid as a model. Front Plant Sci. 2014;5:488.

Barnett MJ, Solow-Cordero DE, Long SR. A high-throughput system to identify inhibitors of Candidatus Liberibacter asiaticus transcription regulators. Proc Natl Acad Sci U S A. 2019;116:18009-14.

Blaustein RA, Lorca GL, Teplitski M. Challenges for managing Candidatus Liberibacter spp. (Huanglongbing disease pathogen): current control measures and future directions. Phytopathology. 2018;108:424-35.

Bové JM. Huanglongbing: a destructive, newly-emerging, century-old disease of citrus. J Plant Pathol. 2006;88(1):7-37.

California Citrus Pest \& Disease Prevention Program. CLas-positive Asian citrus psyllid found in Riverside commercial grove. 2020. https://citrusinsider.org/2 020/08/07/clas-positive-asian-citrus-psyllid-found-in-riverside-commercialgrove.

Canales E, Coll Y, Hernández I, Portieles R, Rodríguez García M, López Y, et al. 'Candidatus Liberibacter asiaticus', causal agent of citrus Huanglongbing, is reduced by treatment with brassinosteroids. PLoS One. 2016:11:e0146223.

Chen Y, Bendix C, Lewis JD. Comparative genomics screen identifies microbeassociated molecular patterns from 'Candidatus Liberibacter' spp. that elicit immune responses in plants. Mol Plant Microbe Interact. 2020;33:539-52.

Clark K, Franco JY, Schwizer S, Pang Z, Hawara E, Liebrand TWH, et al. An effector from the Huanglongbing-associated pathogen targets citrus proteases. Nat Commun. 2018:9:1718.

Clark K, Pang Z, Trinh J, Wang N, Ma W. Sec-delivered effector 1 (SDE1) of Candidatus Liberibacter asiaticus promotes citrus Huanglongbing. Mol Plant Microbe Interact. 2020. https://doi.org/10.1094/MPMI-05-20-0123-R.

Dekkers MGH, Graham JH, Burns JK, Cubero J, Colburn GC. Evaluation of chemical inducers and PR protein reporters for induced systemic resistance to citrus bacterial diseases. (Abstr) Phytopathology. 2004;94:S25.
Deng H, Achor D, Exteberria E, Yu Q, Du D, Stanton D, et al. Phloem regeneration is a mechanism for Huanglongbing-tolerance of "Bearss" lemon and "LB8-9" sugar belle. Front Plant Sci. 2019;10:277.

Duan Y, Zhou L, Hall DG, Li W, Doddapaneni H, Lin H, et al. Complete genome sequence of citrus Huanglongbing bacterium, "Candidatus Liberibacter asiaticus" obtained through metagenomics. Mol Plant Microbe Interact. 2009;22:1011-20.

Durrant WE, Dong X. Systemic acquired resistance. Annu Rev Phytopathol. 2004; 42:185-209.

Frąckowiak P, Pospieszny H, Smiglak M, Obrępalska-Stęplowska A. Assessment of the efficacy and mode of action of benzo (1,2,3)-thiadiazole-7-carbothioic acid S-methyl ester (BTH) and its derivatives in plant protection against viral disease. Int J Mol Sci. 2019;20:1598.

Francis MI, Redondo A, Burns JK, Graham JH. Soil application of imidacloprid and related SAR inducing compounds produces effective and persistent control of citrus canker. Eur J Plant Pathol. 2009;124:283-92.

Gottwald TR, da Graça JV, Bassanezi RB. Citrus Huanglongbing: the pathogen and its impact. Plant Health Progress. 2007. https://doi.org/10.1094/PHP-20070906-01-RV.

Gottwald T, Poole G, McCollum T, Hall D, Hartung J, Bai J, et al. Canine olfactory detection of a vectored phytobacterial pathogen, Liberibacter asiaticus, and integration with disease control. Proc Natl Acad Sci U S A. 2020;117:3492-501.

Gottwald TR. Current epidemiological understanding of citrus Huanglongbing. Annu Rev Phytopathol. 2010;48:119-39.

Gottwald TR, Graham JH, Irey MS, McCollum TG, Wood BW. Inconsequential effect of nutritional treatments on Huanglongbing control, fruit quality, bacterial titer and disease progress. Crop Prot. 2012;36:73-82.

Graham J, Gottwald T, Setamou M. Status of Huanglongbing (HLB) outbreaks in Florida, California and Texas. Trop Plant Pathol. 2020;45:265-78.

Graham JH, Leite RP. Lack of control of citrus canker by induced systemic resistance compounds. Plant Dis. 2004;88:745-50.

Graham JH, Myers ME. Soil application of SAR inducers imidacloprid, thiamethoxam, and acibenzolar-S-methyl for citrus canker control in young grapefruit trees. Plant Dis. 2011;95:725-8.

Graham JH, Myers ME. Integration of soil applied neonicotinoid insecticides and acibenzolar-S-methyl for systemic acquired resistance (SAR) control of citrus canker on young citrus trees. Crop Prot. 2013;54:239-43.

Herman MAB, Restrepo S, Smart CD. Defense gene expression patterns of three SAR-induced tomato cultivars in the field. Physiol Mol Plant Pathol. 2007;71: 192-200.

Hu J, Jiang J, Wang N. Control of Citrus Huanglongbing via trunk injection of plant defense activators and antibiotics. Phytopathology. 2018;108:186-95.

$\mathrm{Hu}$ J, Wang N. Evaluation of the spatiotemporal dynamics of oxytetracycline and its control effect against citrus Huanglongbing via trunk injection. Phytopathology. 2016;106:1495-503.

Hussain S, Rao MJ, Anjum MA, Ejaz S, Umar UU, Ali MA, et al. Effect of different combinations of antibiotics on fruit quality and antioxidant defense system in Huanglongbing infected Kinnow orchards. AMB Express. 2019;9:147.

Jain M, Munoz-Bodnar A, Zhang S, Gabriel DW. A secreted 'Candidatus Liberibacter asiaticus' peroxiredoxin simultaneously suppresses both localized and systemic innate immune responses in planta. Mol Plant Microbe Interact. 2018;31:1312-22

Jia H, Orbović V, Wang N. CRISPR-LbCas12a-mediated modification of citrus. Plant Biotechnol J. 2019;17:1928-37.

Jia H, Wang N. Xcc-facilitated agroinfiltration of citrus leaves: a tool for rapid functional analysis of transgenes in citrus leaves. Plant Cell Rep. 2014;33: 1993-2001.

Jia H, Wang N. Generation of homozygous canker-resistant citrus in the T0 generation using CRISPR-SpCas9p. Plant Biotechnol J. 2020;18:1990-2.

Johnson EG, Wu J, Bright DB, Graham JH. Association of 'Candidatus Liberibacter asiaticus' root infection, but not phloem plugging with root loss on huanglongbing-affected trees prior to appearance of foliar symptoms. Plant Pathol. 2014;63:290-8.

Justyna P-G, Ewa K. Induction of resistance against pathogens by betaaminobutyric acid. Acta Physiol Plant. 2013;35:1735-48.

Kim JS, Wang N. Characterization of copy numbers of 16S rDNA and 16S rRNA of Candidatus Liberibacter asiaticus and the implication in detection in planta using quantitative PCR. BMC Res Notes. 2009;2:37.

Kumagai LB, LeVesque CS, Blomquist CL, Madishetty K, Guo Y, Woods PW, et al. First report of Candidatus Liberibacter asiaticus associated with citrus Huanglongbing in California. Plant Dis. 2013;97:283. 
Li J, Li L, Pang Z, Kolbasov VG, Ehsani R, Carter EW, et al. Developing citrus Huanglongbing (HLB) management strategies based on the severity of symptoms in HLB-endemic citrus-producing regions. Phytopathology. 2019a;109:582-92.

Li J, Pang Z, Duan S, Lee D, Kolbasov VG, Wang N. The in planta effective concentration of oxytetracycline against 'Candidatus Liberibacter asiaticus' for suppression of citrus Huanglongbing. Phytopathology. 2019b;109:2046-54.

Li J, Pang Z, Trivedi P, Zhou X, Ying X, Jia H, et al. 'Candidatus Liberibacter asiaticus' encodes a functional salicylic acid (SA) hydroxylase that degrades SA to suppress plant defenses. Mol Plant Microbe Interact. 2017;30:620-30.

Li J, Trivedi P, Wang N. Field evaluation of plant defense inducers for the control of citrus Huanglongbing. Phytopathology. 2016;106:37-46.

Martinelli F, Uratsu SL, Albrecht U, Reagan RL, Phu ML, Britton M, et al. Transcriptome profiling of citrus fruit response to huanglongbing disease. PLoS One. 2012;7:e38039.

Martínez-Abarca F, Herrera-Cervera JA, Bueno P, Sanjuan J, Bisseling T, Olivares J. Involvement of salicylic acid in the establishment of the Rhizobium melilotialfalfa symbiosis. Mol Plant Microbe Interact. 1998;11:153-5.

Mauch F, Mauch-Mani B, Boller T. Antifungal hydrolases in pea tissue. II. Inhibition of fungal growth by combinations of Chitinase and beta-1,3-glucanase. Plant Physiol. 1988;88:936-42.

Maxson-Stein K, He S-Y, Hammerschmidt R, Jones AL. Effect of treating apple trees with acibenzolar-S-methyl on fire blight and expression of pathogenesis-related protein genes. Plant Dis. 2002:86:785-90.

National Agricultural Statistics Service. Florida citrus statistics 2016-2017: USDA Agricultural Marketing Service; 2018

National Agricultural Statistics Service. Florida citrus statistics 2018-2019: USDA Agricultural Marketing Service; 2020

Nehela Y, Hijaz F, Elzaawely AA, El-Zahaby HM, Killiny N. Citrus phytohormonal response to Candidatus Liberibacter asiaticus and its vector Diaphorina citri. Physiol Mol Plant Pathol. 2018;102:24-35.

Pagliaccia D, Shi J, Pang Z, Hawara E, Clark K, Thapa SP, et al. A pathogen secreted protein as a detection marker for citrus Huanglongbing. Front Microbiol. 2017;8:2041.

Pandey SS, Vasconcelos FNC, Wang N. Spatiotemporal dynamics of Candidatus Liberibacter asiaticus colonization inside citrus plant and Huanglongbing disease development. Phytopathology. 2020. https://doi.org/10.1094/PHYTO09-20-0407-R

Pandey SS, Wang N. Targeted early detection of citrus Huanglongbing causal agent 'Candidatus Liberibacter asiaticus' before symptom expression. Phytopathology. 2019;109:952-9.

Pang Z, Zhang L, Coaker GL, Ma W, He SY, Wang N. Citrus CSACD2 is a target of Candidatus Liberibacter asiaticus in Huanglongbing disease. Plant Physiol. 2020;184:792-805.

Pieterse CMJ, Zamioudis C, Berendsen RL, Weller DM, Van Wees SCM, Bakker PAHM. Induced systemic resistance by beneficial microbes. Annu Rev Phytopathol. 2014;52:347-75.

Pitino M, Allen V, Duan Y. Las $\Delta 5315$ effector induces extreme starch accumulation and chlorosis as $\mathrm{Ca}$. Liberibacter asiaticus infection in Nicotiana benthamiana. Front Plant Sci. 2018;9:113.

Rauscher M, Adam AL, Wirtz S, Guggenheim R, Mendgen K, Deising HB. PR-1 protein inhibits the differentiation of rust infection hyphae in leaves of acquired resistant broad bean. Plant J. 1999;19:625-33.

Rawat N, Kiran SP, Du D, Gmitter FG, Deng Z. Comprehensive meta-analysis, coexpression, and miRNA nested network analysis identifies gene candidates in citrus against Huanglongbing disease. BMC Plant Biol. 2015:15:184.

Riera N, Handique U, Zhang Y, Dewdney MM, Wang N. Characterization of antimicrobial-producing beneficial bacteria isolated from Huanglongbing escape citrus trees. Front Microbiol. 2017;8:2415.

Riera N, Wang H, Li Y, Li J, Pelz-Stelinski K, Wang N. Induced systemic resistance against citrus canker disease by rhizospheric bacteria. Phytopathology. 2018; 108:1038-45

Roka FM, Rouse RE, Muraro RP. Comparison of yields by tree age and rootstock in Southwest Florida orange groves. Proc Fla State Hortic Soc. 2000;113:115-9.

Sétamou M, Alabi OJ, Kunta M, Dale J, da Graça, JV. Distribution of Liberibacter asiaticus in citrus and the Asian citrus psyllid in Texas over a decade. Plant Dis. 2020;104:1118-26.

Silva TR, Valdman E, Valdman B, Leite SGF. Salicylic acid degradation from aqueous solutions using Pseudomonas fluorescens HK44: parameters studies and application tools. Braz J Microbiol. 2007;38:39-44.

Simko I, Piepho HP. The area under the disease progress stairs: calculation, advantage, and application. Phytopathology. 2012;102:381-9.
Singerman A, Burani-Arouca M, Futch $\mathrm{SH}$. The profitability of new citrus plantings in Florida in the era of Huanglongbing. Hortscience. 2018:53(11):1655-63.

Sleiman M, Stawinoga M, Wang S, de Sainte-Claire P, Goupil P, Richard C. Photochemical transformation of the plant activator Acibenzolar-S-methyl in solution. J Photochem Photobiol A: Chem. 2017;333:79-86.

Stansly PA, Arevalo HA, Qureshi JA, Jones MM, Hendricks K, Roberts PD, et al. Vector control and foliar nutrition to maintain economic sustainability of bearing citrus in Florida groves affected by huanglongbing. Pest Manag Sci. 2014;70:415-26.

Tang L, Chhajed S, Vashisth T. Preharvest fruit drop in Huanglongbing-affected Valencia' sweet orange. J Am Soc Hort Sci. 2019;144(2):107-17. https://doi. org/10.21273/JASHS04625-18

Tang L, Vashisth T. New insight in Huanglongbing-associated mature fruit drop in citrus and its link to oxidative stress. Sci Hortic. 2020;265:109246. https://doi. org/10.1016/j.scienta.2020.109246.

Tansey JA, Vanaclocha P, Monzo C, Jones M, Stansly PA. Costs and benefits of insecticide and foliar nutrient applications to huanglongbing-infected citrus trees. Pest Manag Sci. 2017;73(5):904-16.

Thapa N, Danyluk MD, Gerberich KM, Johnson EG, Dewdney MM. Assessment of the effect of thermotherapy on Candidatus Liberibacter asiaticus viability in woody tissue of citrus via graft-based assay and RNA-assay. Phytopathology. 2020a. https://doi.org/10.1094/PHYTO-04-20-0152-R.

Thapa SP, De Francesco A, Trinh J, Gurung FB, Pang Z, Vidalakis G, et al. Genome-wide analyses of Liberibacter species provides insights into evolution, phylogenetic relationships, and virulence factors. Mol Plant Pathol. 2020b;21:716-31.

Trivedi P, Sagaram US, Kim JS, Brlansky RH, Rogers ME, Stelinski LL, et al. Quantification of viable 'Candidatus Liberibacter asiaticus' in hosts using quantitative PCR with the aid of ethidium monoazide (EMA). Eur J Plant Pathol. 2009;124:553-63.

Vallad GE, Goodman RM. Systemic acquired resistance and induced systemic resistance in conventional agriculture. Crop Sci. 2004;44:1920-34.

Vasconcelos FNC, Li J, Pang Z, Vincent C, Wang N. Census of Candidatus Liberibacter asiaticus population inside the phloem of citrus trees. Phytopathology. 2020. https://doi.org/10.1094/PHYTO-09-20-0388-R.

Walters DR, Fountaine JM. Practical application of induced resistance to plant diseases: an appraisal of effectiveness under field conditions. J Agric Sci. 2009;147:523-35

Walters DR, Ratsep J, Havis ND. Controlling crop diseases using induced resistance: challenges for the future. J Exp Bot. 2013;64:1263-80.

Wang N. The citrus huanglongbing crisis and potential solutions. Mol Plant. 2019; 12:607-9.

Wang N. A perspective of citrus Huanglongbing in the context of the Mediterranean Basin. J Plant Pathol. 2020;102:635-40.

Wang N, Pierson EA, Setubal JC, Xu J, Levy JG, Zhang Y, et al. The Candidatus Liberibacter-host interface: insights into pathogenesis mechanisms and disease control. Annu Rev Phytopathol. 2017a;55:451-82.

Wang N, Stelinski LL, Pelz-Stelinski KS, Graham JH, Zhang Y. Tale of the Huanglongbing disease pyramid in the context of the citrus microbiome. Phytopathology. 2017b;107:380-7.

Wang Z, Yin Y, Hu H, Yuan Q, Peng G, Xia Y. Development and application of molecular-based diagnosis for 'Candidatus Liberibacter asiaticus', the causal pathogen of citrus huanglongbing. Plant Pathol. 2006;55:630-8.

Yuan ZC, Edlind MP, Liu P, Saenkham P, Banta LM, Wise AA, et al. The plant signal salicylic acid shuts down expression of the vir regulon and activates quorumquenching genes in Agrobacterium. Proc Natl Acad Sci U S A. 2007;104:11790-5.

Zhang M, Guo Y, Powell CA, Doud MS, Yang C, Duan Y. Effective antibiotics against 'Candidatus Liberibacter asiaticus' in HLB-affected citrus plants identified via the graft-based evaluation. PLoS One. 2014;9:e111032.

Ready to submit your research? Choose BMC and benefit from:

- fast, convenient online submission

- thorough peer review by experienced researchers in your field

- rapid publication on acceptance

- support for research data, including large and complex data types

- gold Open Access which fosters wider collaboration and increased citations

- maximum visibility for your research: over $100 \mathrm{M}$ website views per year

At $\mathrm{BMC}$, research is always in progress.

Learn more biomedcentral.com/submissions 